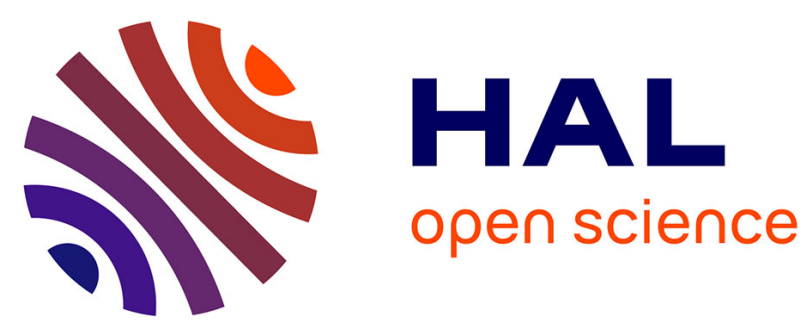

\title{
An approach for establishing the performance maps of the sc-CO2 compressor: Development and qualification by means of CFD simulations
}

Hong-Son Pham, Nicolas Alpy, Jean-Henry Ferrasse, Olivier Boutin, Mark Tothill, Johann Quenaut, Olivier Gastaldi, Thierry Cadiou, Manuel Saez

\section{To cite this version:}

Hong-Son Pham, Nicolas Alpy, Jean-Henry Ferrasse, Olivier Boutin, Mark Tothill, et al.. An approach for establishing the performance maps of the sc-CO2 compressor: Development and qualification by means of CFD simulations. International Journal of Heat and Fluid Flow, 2016, 61 (Part B), pp.379394. 10.1016/j.ijheatfluidflow.2016.05.017 . hal-01461787

\section{HAL Id: hal-01461787 \\ https://hal.science/hal-01461787}

Submitted on 18 Apr 2018

HAL is a multi-disciplinary open access archive for the deposit and dissemination of scientific research documents, whether they are published or not. The documents may come from teaching and research institutions in France or abroad, or from public or private research centers.
L'archive ouverte pluridisciplinaire HAL, est destinée au dépôt et à la diffusion de documents scientifiques de niveau recherche, publiés ou non, émanant des établissements d'enseignement et de recherche français ou étrangers, des laboratoires publics ou privés. 


\title{
An approach for establishing the performance maps of the $\mathrm{sc}-\mathrm{CO}_{2}$ compressor: development and qualification by means of CFD simulations
}

\author{
H.S. Pham ${ }^{1}$, N. Alpy ${ }^{1}$, J.H. Ferrasse ${ }^{2}$, O. Boutin ${ }^{2}$, M. Tothill ${ }^{3}$, J. Quenaut ${ }^{3}$, O. Gastaldi ${ }^{4}$, T. Cadiou ${ }^{1}$, M. Saez ${ }^{1}$ \\ ${ }^{1}$ CEA Cadarache, DEN/DER/SESI/LEMS, FR-13108 Saint-Paul-lez-Durance, France \\ hong-son.pham@cea.fr; nicolas.alpy@cea.fr; thierry.cadiou@cea.fr; manuel.saez@cea.fr; \\ ${ }^{2}$ Aix-Marseille Université, CNRS, ECM, M2P2 UMR 7340, FR-13451 Marseille, France \\ jean-henry.ferrasse@univ-amu.fr; olivier.boutin@univ-amu.fr \\ ${ }^{3}$ Alstom, Brown Boveri Strasse 7,CH-5401 Baden,Switzerland \\ johann.quenaut@power.alstom.com;mark.tothill@power.alstom.com \\ ${ }^{4}$ CEA Cadarache, DEN/DTN, FR-13108 Saint-Paul-lez-Durance, France \\ olivier.gastaldi@cea.fr
}

\section{Highlights}

- Ability of CFD to predict the performance of a sc- $\mathrm{CO}_{2}$ test compressor is shown.

- Risk of vapor pockets occurrence inside a scale 1:1 compressor is highlighted.

- Limitation of previous performance maps approaches to model real gas behavior is shown.

- A performance maps approach for sc- $\mathrm{CO}_{2}$ compressor is proposed and validated.

\begin{abstract}
One of the challenges in the performance prediction of the supercritical $\mathrm{CO}_{2}\left(\mathrm{sc}-\mathrm{CO}_{2}\right)$ compressor is the real gas behavior of the working fluid near the critical point. This study deals with the establishment of an approach that allows coping with this particularity by dressing compressor performance maps in adequate reduced coordinates (i.e. suitable dimensionless speed and flow parameters inputs and pressure ratio and enthalpy rise outputs), while using CFD for its validation. Two centrifugal compressor designs have been considered in this work. The first one corresponds to a $6 \mathrm{~kW}$ small scale component implemented in a test loop at Tokyo Institute of Technology. The second one corresponds to a $38 \mathrm{MW}$ scale 1:1 design considered at an early stage of a project that investigates $\mathrm{sc}-\mathrm{CO}_{2}$ cycle for a Small Modular Reactor application. Numerical results on the former have been successfully confronted with the experimental data to qualify the ability of CFD to provide a performance database. Results on the latter have revealed a significant decrease in the static temperature and pressure during flow acceleration along the leading edge of the impeller blades. In this line, the increased risk of vapor pockets appearance inside a $\mathrm{sc}-\mathrm{CO}_{2}$ compressor has been highlighted and recommendations regarding the choice of the on-design inlet conditions and the compressor design have been given to overcome this concern. CFD results on the scale $1: 1$ compressor have then been used to evaluate the relevancy of some previous performance maps approaches for a sc- $\mathrm{CO}_{2}$ compressor application. These include the conventional approach for ideal gas and its derivation, as well as a reference approach from the literature that was previously applied to model an sc- $\mathrm{CO}_{2}$ test compressor. As the dimensionless parameters of these approaches are found to yield discrepancies on the compressor performance, a revised approach that incorporates real gas formulations into turbomachinery key similarity parameters has been finally proposed. In support, an extensive number of CFD case studies has been carried out at various compressor inlet conditions, providing numerical results for its qualification. Accordingly, the proposed approach has been found to succeed in consistently representing and accurately predicting the $\mathrm{sc}-\mathrm{CO}_{2}$ compressor performance over a wide operating range.
\end{abstract}

\section{Keywords}

Supercritical $\mathrm{CO}_{2}$, compressor, CFD, performance maps, critical point 


\begin{tabular}{llll}
\hline Nomenclature & \multicolumn{2}{l}{ Greek symbols } \\
\hline$a$ & Speed of sound $(\mathrm{m} / \mathrm{s})$ & $\Delta H$ & Total enthalpy rise $(\mathrm{kJ} / \mathrm{kg})$ \\
$E k$ & Eckert number & $\gamma$ & Specific heat ratio \\
$E u$ & Euler number & $\eta$ & Efficiency \\
$k_{v}$ & A similarity factor & $\Pi$ & Pressure ratio \\
$M$ & Mach number & $\rho$ & Density $\left(\mathrm{kg} / \mathrm{m}^{3}\right)$ \\
$\dot{m}$ & Mass flowrate $(\mathrm{kg} / \mathrm{s})$ & \multicolumn{2}{l}{} \\
\cline { 2 - 3 }$N$ & Shaft speed $(\mathrm{rpm})$ & \multicolumn{2}{l}{ Subscripts } \\
\cline { 2 - 3 }$n_{s}$ & Isentropic volume exponent & $a$ & Actual conditions or values \\
$P$ & Pressure $(\mathrm{MPa})$ & $a d$ & Dimensionless parameter \\
$r$ & Gas constant $\left(\mathrm{J} / \mathrm{kg} .{ }^{\circ} \mathrm{C}\right)$ & $c$ & Critical point (for pressure or temperature) \\
$R e$ & Reynolds number & $c$ & Corrected values \\
$s$ & Specific entropy $\left(\mathrm{J} / \mathrm{kg} .{ }^{\circ} \mathrm{C}\right)$ & $c r$ & Critical (i.e. sonic) conditions \\
$T$ & Temperature $\left({ }^{\circ} \mathrm{C}\right)$ & in & Compressor inlet \\
$V$ & Velocity $(\mathrm{m} / \mathrm{s})$ & out & Compressor inlet \\
$v$ & Specific volume $\left(\mathrm{m}^{3} / \mathrm{kg}\right)$ & $p c$ & Pseudocritical point \\
$Z$ & Compressibility factor & $r e f$ & Reference values \\
\hline
\end{tabular}

\section{Introduction}

The supercritical $\mathrm{CO}_{2}\left(\mathrm{sc}-\mathrm{CO}_{2}\right)$ cycle is viewed as a potential candidate for advanced power conversion systems due to the following advantages (Dostal, 2004): (i) potentially high efficiency thanks to the low compression work in the reduced compressibility region near critical point; (ii) smaller size of the turbomachinery; (iii) simpler system layout. Interest in the $\mathrm{sc}-\mathrm{CO}_{2}$ cycle can be seen through an increasing number of works that have been conducted over the last decade in view of analyzing its advantages for various heat sources. Typical applications of the $\mathrm{sc}-\mathrm{CO}_{2}$ cycle are low grade waste heat applications (Chen, 2011), hybrid fuel cell systems (Sanchez et al., 2009), concentrated solar power plants (Turchi et al., 2013), nuclear reactors including innovative small modular reactors (Sienicki, 2005; Yoon et al., 2012), next generation nuclear reactors (Dostal, 2004; Hejzlar et al., 2006; Floyd et al., 2013) and fusion reactor concepts (Ishiyama et al., 2008). An overview on the performance of the $\mathrm{sc}-\mathrm{CO}_{2}$ cycle in a large range of heat source temperature can be found in (Pham et al., 2015).

The reduced work of the sc- $\mathrm{CO}_{2}$ compressor takes advantage of the real gas behavior of the working fluid near the critical point. However, the sharp change of fluid properties in this region can be viewed as having possible implications on the stable operation of the compressor and thus of the cycle. Besides, the real gas behavior also makes very questionable the application of the know-how gained on conventional turbomachines to the sc- $\mathrm{CO}_{2}$ compressor. For instance, Wright et al. (2010) admitted that their early attempts to use existing design tools and to couple them to the NIST REFPROP database for sc- $\mathrm{CO}_{2}$ compressor design were unsuccessful. They suggested that it was probably due to the fact that formulations and solution methods used by the available design tools were based on the specific heat capacity, which was no longer a relevant indicator for the enthalpy change of sc- $\mathrm{CO}_{2}$.

In order to investigate the performance and operation of the $\mathrm{sc}-\mathrm{CO}_{2}$ compressor near the critical point, test loops were built at Tokyo Institute of Technology (TiTech) and Sandia National Laboratories (SNL). Tests at TiTech were said to confirm the stable operation of the compressor near the critical point and the reduced compression work in the supercritical region (Aritomi et al., 
2011). Tests at SNL were reported to demonstrate the capability of operating the sc- $\mathrm{CO}_{2}$ compressor in the vapor, liquid, and supercritical regions (Wright et al., 2010). In addition, compressor operation in the two-phase region was observed to yield no dysfunctional behavior (Noall and Pasch, 2014).

In parallel with the collection of experimental data on the $\mathrm{sc}-\mathrm{CO}_{2}$ test loops at TiTech and SNL, significant efforts have been devoted to the design and performance prediction of the $\mathrm{sc}-\mathrm{CO}_{2}$ compressor through both 1D analysis (meanline code) and CFD simulation. Meanline codes as those reported in (Moisseytsev and Sienicki, 2010; Vilim, 2010) are based on the classical approach of establishing the one-dimensional mass, momentum and energy conservation equations for the compressor. It is important to note that these codes represent different loss terms using empirical correlations in the literature whose relevant application for the $\mathrm{sc}-\mathrm{CO}_{2}$ compressor is still an issue.

CFD simulations have been performed on the sc- $\mathrm{CO}_{2}$ compressor used in the test loops, providing results to confront with the experimental data. Aritomi et al. (2011) investigated the TiTech compressor using the commercial code CFX. Thermodynamic properties of $\mathrm{CO}_{2}$ were obtained using the Aungier-Redlich-Kwong equation of state. Turbulence modeling was assured through the $\mathrm{k}-\varepsilon$ model. It was then concluded that the calculated compressor outlet temperature and pressure coincided well with the experimental data. The maximum discrepancy for the temperature and pressure were $0.14 \mathrm{MPa}$ and $0.4{ }^{\circ} \mathrm{C}$, respectively. Rinaldi et al. (2013) used a parallel solver for the solution of Navier-Stokes equations on unstructured meshes to study the sc$\mathrm{CO}_{2}$ compressor mounted on the SNL test loop. Fluid properties were interpolated from a precomputed look-up table that was generated form the NIST database. The k- $\omega$ SST turbulence model was employed. Compared to the experimental data, the efficiency and head coefficient resulted from the simulation showed a relative difference of about $4 \%$ and $25 \%$, respectively.

The use of different physical models, typically the turbulence model, in these early investigations has not been sufficiently backed due to the lack of relevant knowledge. Literature on this topic can only be found in the study of heat transfer problem in supercritical fluid flows. Accordingly, Hiroaki et al. (1973) suggested that the sharp variation of thermal physical properties might change the velocity profile, and thus the shear stress distribution and turbulence production. In this case, various turbulence models available in the simulation codes are no longer relevant to be used for supercritical fluid flows. In the adiabatic case, nevertheless, experiments showed that the flow structure and the near-wall velocity profile in supercritical flows remained similar to those in liquid and gas flows (Kurganov and Kaptil'ny, 1992). As such, commonly used turbulence models can be expected to not yield significant irrelevancy when applied to the $\mathrm{sc}-\mathrm{CO}_{2}$ flow in the compressor.

Performance analysis through these numerical tools shows its advantages for preliminary sizing, design and operation validation of turbomachinery. However, this approach is relatively expensive for application to the turbomachines integrated in a complex system such as an energy conversion system, especially for transient studies. In such cases, turbomachine performance maps are used to provide the performance parameters, conventionally pressure ratio and efficiency. These latter are indeed calculated/interpolated through explicit functions of corrected mass flowrate and shaft speed, thereby speeding up the calculation. The corrected parameters can be derived from the scale analysis on the basis of the similarity conditions. They allow taking into account the departure of the compressor operating conditions from the reference conditions at which the maps are generated. It is important to note that the development of these parameters incorporates the ideal gas assumptions. Specifically, the working fluid in the turbomachines must follow the ideal gas law and feature a constant specific heat ratio.

Moisseytsev and Sienicki (2006), using a meanline code, showed that a small change in inlet pressure or inlet temperature caused a significant impact on the compressor performance. As such, the conventional corrected shaft speed and mass flowrate were seen to be not able to represent these effects. An alternative approach that involves generation of performance maps for individual variations of each of the operating conditions was therefore proposed. These four- 
dimensional maps provide the mass flow rate and outlet temperature resulting from the mean line code at a given set of shaft speed, inlet temperature, inlet pressure, and outlet pressure. Note that such an approach, though pragmatic, could be seen as increasing the experimental effort of validation and making more complex the numerical treatment of performance maps in a system code.

Another approach of sc- $\mathrm{CO}_{2}$ compressor performance maps was provided by BNI (BarberNichols Inc.) on the basis of the similarity conditions (Wright et al., 2011). As the conventional approach for ideal gas turbomachinery, the BNI approach consists in correcting the mass flow rate and the shaft speed with respect to the compressor inlet conditions. These corrections, which are based on formulations given in (Glassman, 1972) for non-ideal gas compressors, refer to the critical conditions (i.e. sonic conditions) instead of the commonly used total conditions (i.e. stagnation conditions). The choice of the critical conditions was previously explained by the fact that it allows taking into account the change of specific heat ratio (Glassman, 1972). As such, the $\mathrm{BNI}$ approach is expected to be able to consistently represent the performance of the $\mathrm{sc}-\mathrm{CO}_{2}$ compressor. Tests performed at SNL provided data to establish the performance maps of the sc$\mathrm{CO}_{2}$ compressor in comparison with the $\mathrm{BNI}$ prediction. The measured data in the supercritical region were reported to be in agreement with the predicted values (Wright et al., 2010). Though, test data in the liquid phase revealed larger discrepancies compared to those in the supercritical region (Wright et al., 2011).

The present study investigates the establishment of the $\mathrm{sc}-\mathrm{CO}_{2}$ compressor performance maps by mean of CFD simulations. These latter are performed using the commercial code STAR$\mathrm{CCM}+$. First, the geometrical and physical models of the $\mathrm{sc}-\mathrm{CO}_{2}$ centrifugal compressor are presented. Then, simulation results on the small scale TiTech compressor are reported in comparison with the experimental data to qualify the CFD technique. Next, some particularities in the compression of $\mathrm{sc}-\mathrm{CO}_{2}$ are disclosed thanks to the simulations on a full scale compressor design, providing feedbacks for future $\mathrm{sc}-\mathrm{CO}_{2}$ compressor sizing. Finally, limitations of previous performance maps approaches for the $\mathrm{sc}-\mathrm{CO}_{2}$ compressor are highlighted and a new approach is proposed to cope with the real gas behavior of $\mathrm{sc}-\mathrm{CO}_{2}$, such as compressibility. This is qualified through CFD results to assess its ability to consistently represent and reliably predict the $\mathrm{sc}-\mathrm{CO}_{2}$ compressor performance.

\section{CFD simulations of the $\mathrm{sc}-\mathrm{CO}_{2}$ compressors}

\subsection{Sc- $\mathrm{CO}_{2}$ centrifugal compressor modeling}

\subsubsection{Geometrical modeling and boundary conditions}

\subsubsection{Compressor models}

Two centrifugal sc- $\mathrm{CO}_{2}$ compressors are considered for the CFD simulations: a small scale one used in the TiTech test loop and a full scale one, named as SMR compressor, which is considered at an early stage of a project that investigates $\mathrm{sc}-\mathrm{CO}_{2}$ cycle coupling to a Small Modular Reactor (Pham et al., 2015). The small scale TiTech compressor has a vane diffuser while the full scale one is designed with a vaneless diffuser. Both impellers are unshrouded and have backward curved blades (i.e. the impellers spin clockwise in Fig 1). Design conditions and main dimensions of both TiTech and SMR compressors are recapitulated in Table 1, together with those of the small scale SNL compressor, used in the SNL test loop, for reference. Pictures of these compressors are given in Fig 1. 

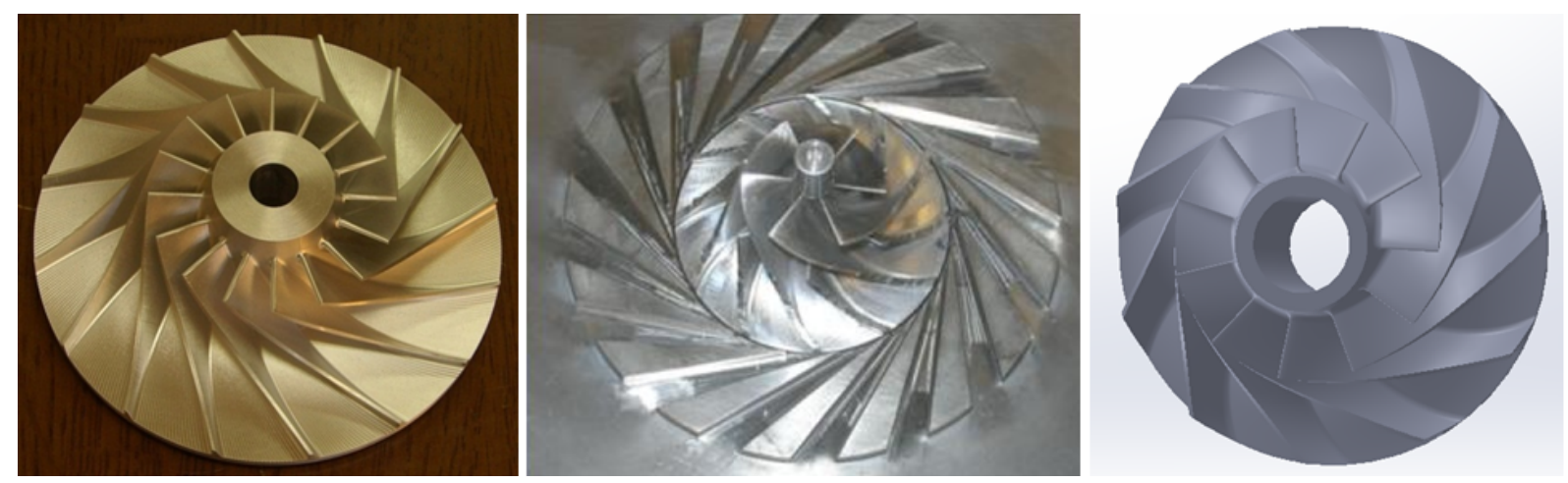

Fig 1: Design of TiTech, SNL and SMR compressor (from left to right).

Table 1: Sc- $\mathrm{CO}_{2}$ compressor design conditions and main dimensions.

\begin{tabular}{|l|c|c|c|}
\hline & TiTech & SNL & SMR \\
\hline Scale & small & small & $1: 1$ \\
\hline Power, kWe & 6 & 50 & 38340 \\
\hline Pressure ratio & 1.19 & 1.8 & 2.38 \\
\hline Mass flow rate, kg/s & 2.0 & 3.5 & 2324 \\
\hline Shaft speed, rpm & 24000 & 75000 & 7000 \\
\hline Impeller exit diameter, mm & 76 & 37.4 & 498 \\
\hline Impeller-tip inlet diameter, mm & 40 & 18.7 & 289 \\
\hline Impeller-hub inlet diameter, mm & 19.97 & 5.1 & 144 \\
\hline Outflow depth, mm & 3.4 & 1.7 & 38 \\
\hline Clearance gap, mm & 0.20 & 0.25 & $\mathrm{n} / \mathrm{a}$ \\
\hline Number of impeller blades & 16 & $6 / 6$ & 11 \\
\hline Number of diffuser blades & 19 & 17 & 0 \\
\hline
\end{tabular}

\subsubsection{Fluid flow models and mesh}

The calculation domain, i.e. the region occupied by the fluid flow, can be generated from the CAD model of compressor. Note that for an axisymmetric problem such as the flow in the compressor, a simplified domain can be used to significantly reduce the computational cost. For the TiTech compressor, only one-sixteenth of the impeller and one-nineteenth of the vane diffuser are modeled, in line with the number of blades of impeller (16) and diffuser (19) (cf. Fig 2). Similarly, only one-eleventh of the impeller and of the vaneless diffuser is modeled for the SMR compressor (cf. Fig 3). Periodic boundaries will be then adopted to assure the continuity of the fluid flow. The tip clearance is taken into account for the TiTech compressor but is neglected for the SMR compressor. Indeed, the tip clearance has more important effect on the performance of the small scale compressor, compared to that of the full scale one. 


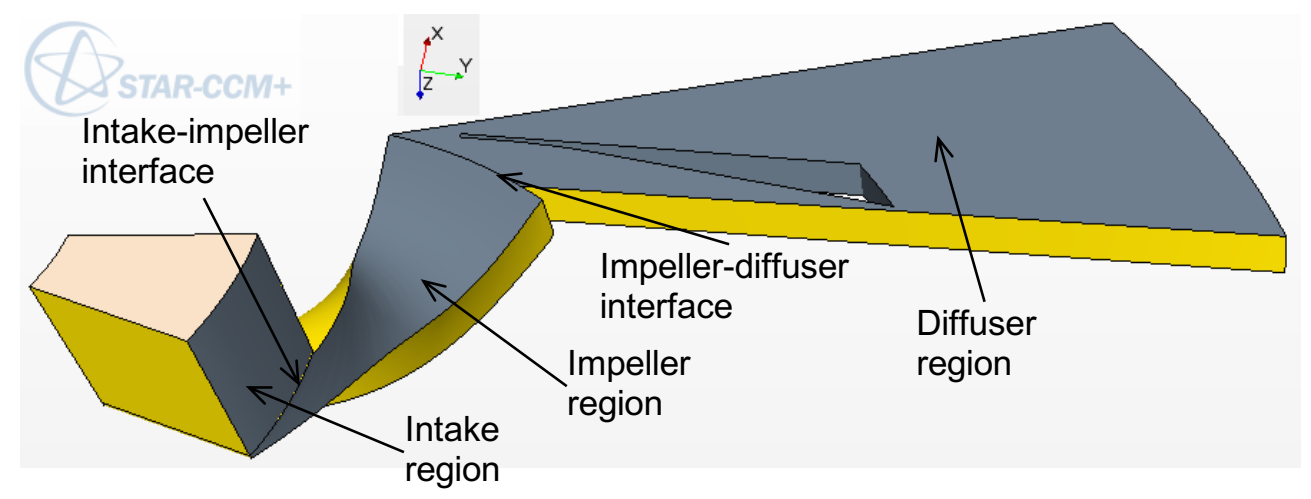

Fig 2: Calculation domain for the TiTech compressor simulation. Impeller blade is hidden under the tip-clearance flow.

For the TiTech compressor, the simulation domain ends up with the diffuser, i.e. the volute being excluded. Parenthetically, a reason for omitting the volute in TiTech compressor is the difficulty faced when trying to interpret its $3 \mathrm{D}$ shape in the component drawing. Nevertheless, this volute has very small effect in the change of the static pressure at the compressor outlet due to the low velocity of the fluid leaving the diffuser. This is not the case of the full scale SMR compressor, for which a symmetric two-outflow volute was designed to efficiently slow down the fluid flow at the diffuser outlet, providing a complementary static pressure increase. The fluid region in the volute can be modeled as a half of the real one as in Fig 3, using the periodic boundary conditions. Finally, an intake region is also added to the upstream of the impeller region to avoid the perturbations of the rotating effects on the inlet flow. Table 2 recapitulates the simplification of the fluid flow region for both TiTech and SMR compressors.

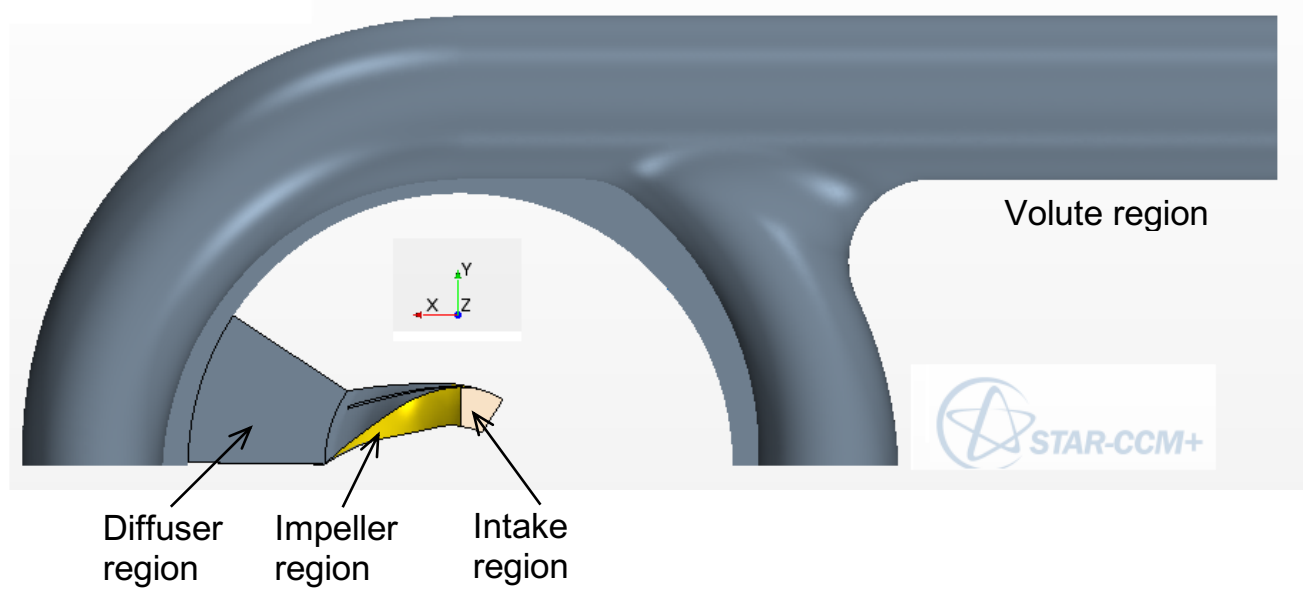

Fig 3: Calculation domain for the SMR compressor modeling.

Table 2: Summary of fluid flow domain simplification for the TiTech and SMR compressor modeling.

\begin{tabular}{|l|c|c|}
\hline Fluid region & TiTech & SMR \\
\hline Intake & $1 / 16$ & $1 / 11$ \\
\hline Impeller & $1 / 16$ & $1 / 11$ \\
\hline Diffuser & $1 / 19$ & $1 / 11$ \\
\hline Volute & N/A & $1 / 2$ \\
\hline
\end{tabular}




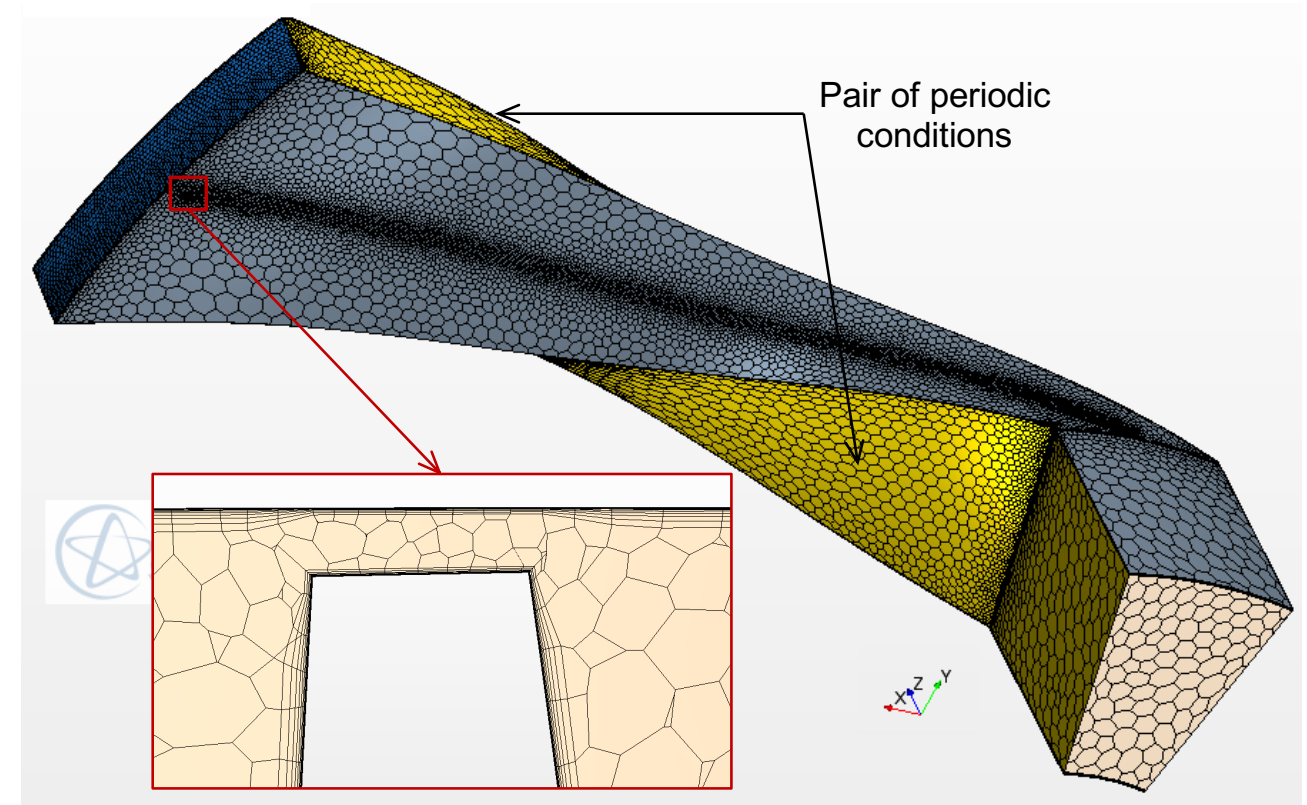

Fig 4: TiTech compressor grid with a zoom on tip clearance refinement. Periodic conditions are applied for pairs of lateral surfaces.

Polyhedral mesh is generated for the fluid flow domain of each compressor (cf. Fig 4 and Fig 5). The total numbers of cells for the fluid flow domain of the TiTech and SMR compressor are around 250000 and 500 000, respectively. Note that a special attention is placed on the boundary layer region where prism layers are used to control the position of the first cell near the solid wall. This is to keep the wall distance in the viscous length coordinate (wall $y^{+}$) around $30-120$, which is indeed the recommended range for using the wall function approach to solve the turbulence properties in the near wall region. There are two reasons for such a choice. First, the case studies featuring large incidence angles at impeller inlet are not engaged in this work and thus flow separation is avoided; the wall-function approach can therefore provide sufficiently accurate results. Second, this approach is privileged to reduce the computational cost since an extensive number of simulations is envisaged to establish the compressor performance maps.

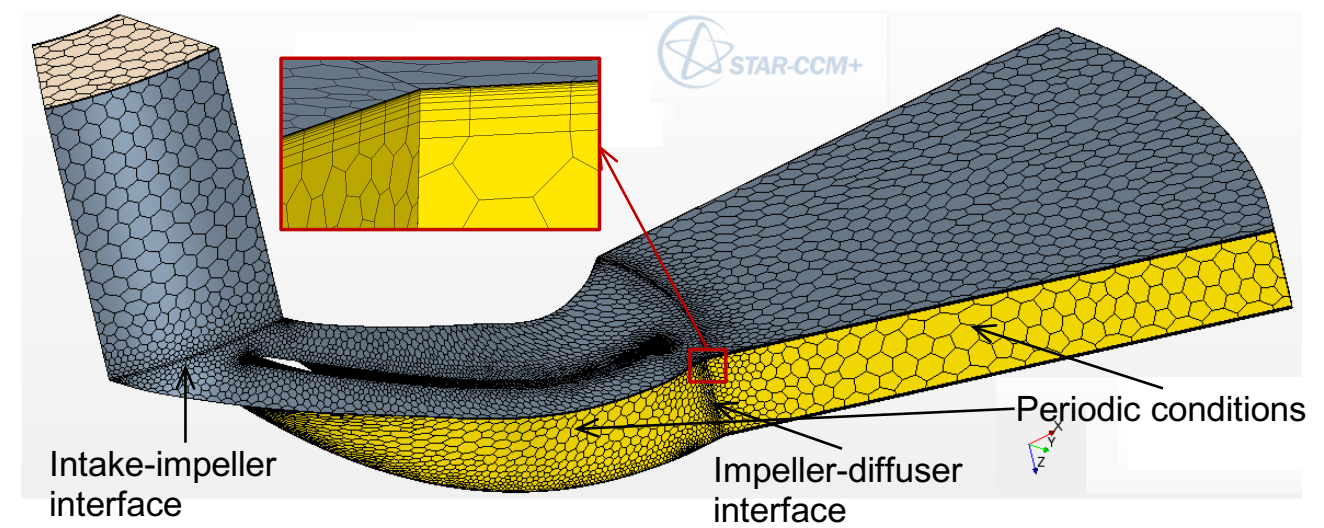

Fig 5: SMR compressor grid with prism layers in the near-wall regions. Mixing-plan interfaces are defined at the intersection of two regions. Periodic conditions are applied for pairs of lateral surfaces.

\subsubsection{Boundary conditions setup}

For the CFD simulations in STAR-CCM+, boundary conditions such as temperature and mass flowrate at the compressor inlet and pressure at the compressor outlet should be specified to the solver. The shaft speed should also be defined as an input parameter for the calculation. The outlet temperature and inlet pressure will be obtained as the simulation results. Additionally, the shaft torque can be exported from the solution to derive the compressor performance. These conditions 
are summarized in Table 3. The periodic conditions are defined for different pairs of surfaces as mentioned above. The remaining surfaces, including the hub and shroud surfaces as well as the wall surfaces of impeller and diffuser blades, are specified as non-slip adiabatic wall.

Table 3: CFD calculation input and output data.

\begin{tabular}{|l|c|c|}
\hline Compressor conditions & CFD input & CFD output \\
\hline Inlet temperature & $\bullet$ & \\
\hline Inlet pressure & & $\bullet$ \\
\hline Outlet temperature & & $\bullet$ \\
\hline Outlet pressure & $\bullet$ & \\
\hline Mass flow rate & $\bullet$ & \\
\hline Shaft speed & $\bullet$ & \\
\hline Torque & & $\bullet$ \\
\hline
\end{tabular}

As indicated above, it is the compressor outlet pressure which is set for the calculation and the compressor inlet pressure is a result in STAR-CCM+. However, compressor performance analysis is commonly performed at given inlet conditions, letting outlet conditions as the results. Indeed, this practice is convenient for generating the compressor performance maps since the flow and speed parameters should be predetermined through the inlet conditions. For the mentioned purpose, a java program was developed and run simultaneously with the STAR-CCM+ calculation: the compressor outlet pressure is automatically adjusted during the iterative process in order to achieve a preset compressor inlet pressure.

\subsubsection{Physic modelling}

\subsubsection{Modeling of the fluid properties}

The free Helmholtz energy equation proposed by Span and Wagner (1996) is used to accurately predict the $\mathrm{CO}_{2}$ thermodynamic properties in the near critical region. This equation of state is, unfortunately, not available in most of commercial CFD codes. Nevertheless, STAR-CCM+ provides the possibility to implement an equation of state throughout field functions or look-up tables for thermodynamic properties of fluid. During the simulation process, these field functions are called or an interpolated lookup algorithm is used to compute the required properties at a given set of state variables, specifically temperature and pressure. The method using look-up tables is quite simple and is therefore adopted in this work.

The ranges of the lookup tables have been chosen in $\left[-30 ; 90{ }^{\circ} \mathrm{C}\right]$ for temperature and $[1.5 ; 30$ $\mathrm{MPa}$ ] for pressure in view of covering the compression line when operating the compressor in the liquid and supercritical regions. Three tables are defined for the density, enthalpy, and speed of sound, as required in STAR-CCM+. The range of the look-up table mentioned above includes not only the supercritical phase but also the liquid phase. This is our intention to perform the simulations of the sc- $\mathrm{CO}_{2}$ compressors at various inlet conditions, even close to the saturation dome. In this latter case, the temporary solution of the iterative process (i.e. on-going converged solution) may have a local region that drifts into the two-phase condition. As such, the discontinuities in the fluid thermodynamic properties throughout the liquid-vapor coexistence curve will lead to long-time iterative process or even non-converged calculation. 

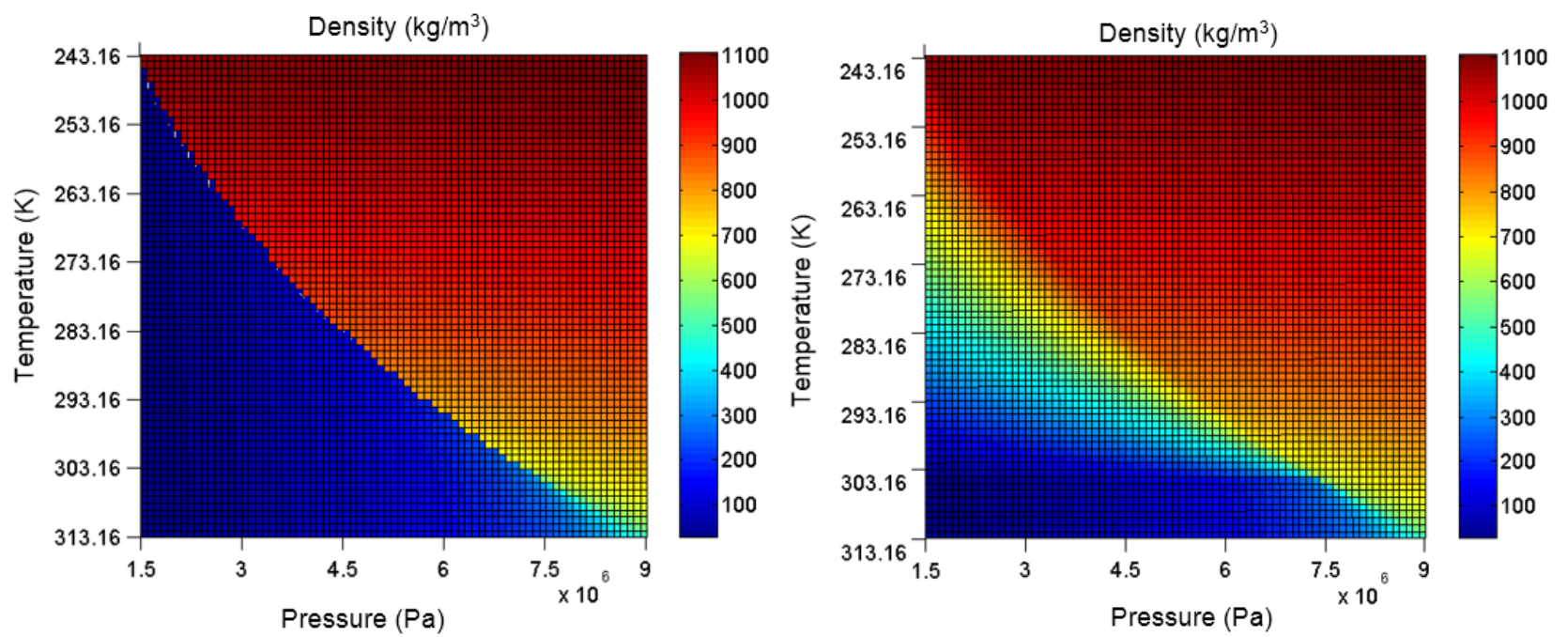

Fig 6: Discontinuity in the $\mathrm{CO}_{2}$ density across the saturation curve from the equation of state (left) and the modified equation for numerical purposes with a continuity in the density across the saturation curve (right).

To overcome this concern, the properties of $\mathrm{CO}_{2}$ such as density, enthalpy, and speed of sound in the vapor region next to the saturation line are replaced by those interpolated from the liquid and the remaining vapor region. This approach is illustrated in Fig 6 for the $\mathrm{CO}_{2}$ density. Similar technique has been extensively used for calculating metastable properties of steam used in the IPAWS-97 database and recently used for calculating subcooled vapor of $\mathrm{CO}_{2}$ in (Baltadjiev et al., 2014). This latter study extrapolates the vapor properties to the liquid region near the saturation curve to study the effect of local condensation during the compression of $\mathrm{CO}_{2}$ from the vapor region. In the present study, however, this technique is used only for the numerical purposes. As the fluid properties continuity is ensured artificially across the saturation curve, there is no numerical problem encountered during the calculation. The final solution can be easily checked to see whether there exists a two-phase zone inside the fluid domain, by simply comparing the minimum pressure with the saturation pressure. If the on-set of the two-phase condition is detected, the calculation results should be discarded since the physical meaning of such an approach is not ensured.

\subsubsection{Modeling of the fluid flow}

The operation of a centrifugal compressor is inherently an unsteady process, i.e. the flow present in the centrifugal compressor is unsteady. This is mainly a result of the aerodynamic interaction between the rotating and stationary parts. Hence, the modeling of turbomachinery flows involves the problem of handling the rotating effects and the rotor-stator interaction. In spite of the unsteady nature in the centrifugal compressor flow, it can be modeled as a steady-state problem using the so-called multiple frames of reference approach, together with a mixing-plane interface. This technique consists in using a moving frame and accounting for additional terms for the Coriolis and centrifugal forces. The flow in the rotating component, such as the impeller, is modeled in a rotating frame of reference whereas the remaining flow in the stationary parts can be, as usual, solved with equations in the stationary frame. Further details on this approach, as well as on the other ones for turbomachinery flow modeling, can be found in (Liu and Hill, 2000).

As previously discussed, commonly used turbulence models can be expected to be used for modeling the $\mathrm{sc}-\mathrm{CO}_{2}$ compressor. Even with this assumption, selecting a suitable turbulence model for turbomachinery simulations always remains a challenging task. Turbulence models need to be able to cope with high Reynolds number flows as well as complex flow dynamics inside the turbomachinery. The qualification of turbulence model can only be done with sufficient various experimental data which range from turbulence properties to mean flow properties as well as data related to the turbomachine performance. Such detailed information is not available and thus it cannot be the objective of this work to come toward the selection of a most relevant turbulence model. Besides, the impact of turbulence modeling on the compressor performance could be seen 
as secondary effects in comparison with the ones resulting from the real gas behavior of the working fluid (e.g. variation of the fluid compressibility), which is focused on in this study. In that context, the realizable $k-\varepsilon$ model is conservatively used as it is more proven for a variety of types of flows (Karthik, 2011).

\subsection{Simulation results of the $\mathrm{Sc}-\mathrm{CO}_{2}$ compressors}

The on-design inlet conditions of the three $\mathrm{sc}-\mathrm{CO}_{2}$ compressors mentioned in the previous chapter are recapitulated in Table 4. The SNL compressor (shown therein for reference) operates entirely in the supercritical region with the compression starting from a point very close to the critical point. The TiTech compressor, on the other hand, was designed with subcritical gas condition at the inlet and supercritical condition at the outlet. The full scale SMR machine compresses $\mathrm{CO}_{2}$ in the subcritical liquid condition and discharges $\mathrm{sc}-\mathrm{CO}_{2}$ at the outlet. The ondesign inlet and outlet conditions of each compressor are illustrated in Fig 7. Although phase change occurs inside both TiTech and SMR compressors, these transitions take place between the regions where fluid properties feature no abrupt variation (i.e. from subcritical liquid to supercritical region or from subcritical gas to supercritical region).

Table 4: Comparison of the sc- $\mathrm{CO}_{2}$ compressors on-design inlet conditions.

\begin{tabular}{|l|c|c|c|}
\hline Fluid phase & $\begin{array}{c}\text { TiTech } \\
\text { Subcritical } \\
\text { gas }\end{array}$ & Supercritical & $\begin{array}{c}\text { Subcritical } \\
\text { liquid }\end{array}$ \\
\hline Temperature, ${ }^{\circ} \mathrm{C}$ & 32 & 32 & 27 \\
\hline Pressure, $\mathrm{MPa}$ & 6.80 & 7.70 & 8.4 \\
\hline Density, $\mathrm{kg} / \mathrm{m}^{3}$ & 220 & 587 & 758 \\
\hline Compressibility & 0.53 & 0.22 & 0.19 \\
\hline
\end{tabular}

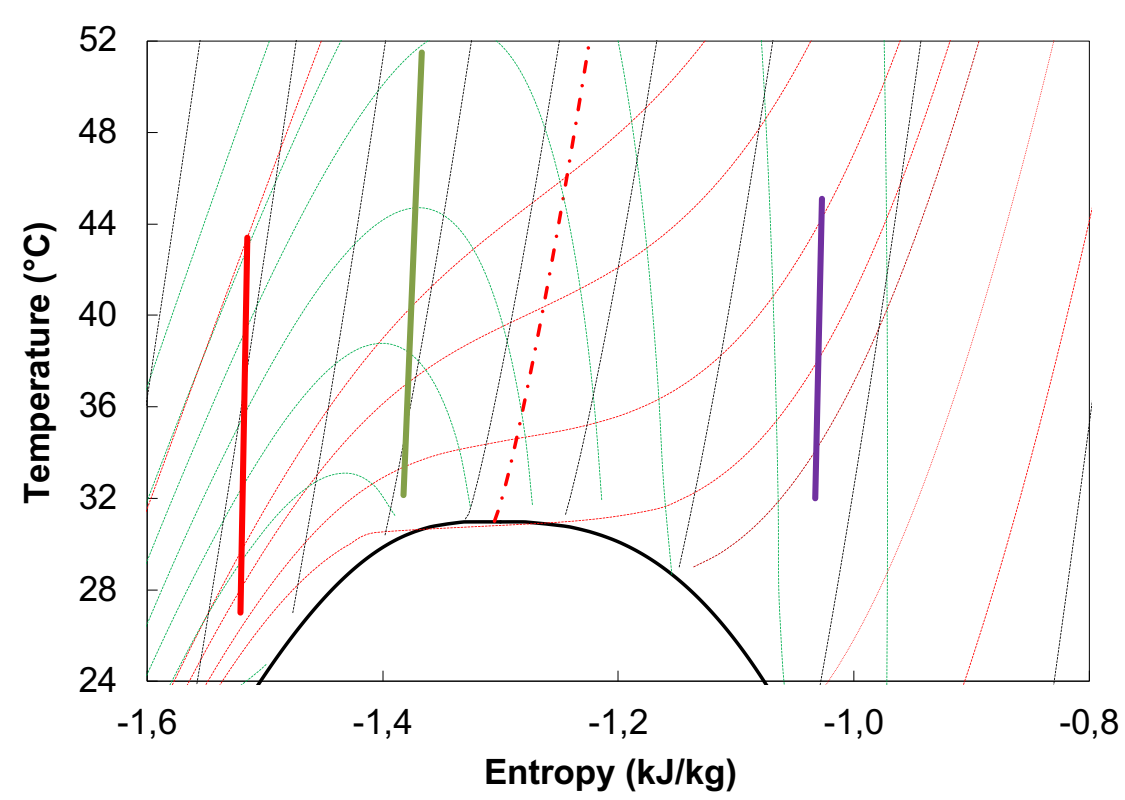

Fig 7: On-design inlet and outlet conditions of different sc- $\mathrm{CO}_{2}$ compressors with various isobar, iso-compressibility, and iso-density lines. $T_{c}, P_{c t}$ and $P_{p c}$ denote the critical temperature, critical pressure and pseudocritical pressure, respectively.

\subsubsection{Simulation results on the small scale compressor}

TiTech compressor simulations have been performed at the on-design conditions and elsewhere. Among available experimental data, those with inlet conditions located at very different areas, typically in terms of compressibility, have been selected for off-design simulations. Though, there is only one point available in the liquid-like supercritical region (i.e. at high compressor inlet 
density). The remaining points are either in the subcritical gas phase or gas-like supercritical phase.

The temperature and pressure contours on the impeller blade wall and hub surface are shown in Fig 8. The continuity in the evolution of these parameters along the fluid flow domain allows illustrating the achievement of a converged solution. The compressor outlet conditions resulted from the CFD simulations are shown in the T-s diagram in Fig 9, together with the inlet and outlet conditions monitored on the test loop. The digital data are reported in Table 5 to ease discussion. Overall, there is a good agreement between the experimental data and the simulation results regarding the compressor outlet temperature and pressure. The discrepancies are roughly limited below the sensor uncertainties, $0.50{ }^{\circ} \mathrm{C}$ for temperature and $0.020 \mathrm{MPa}$ for pressure. From the T-s diagram, it comes out that the unique set of operating conditions in the liquid-like supercritical region leads to an entropy decrease along the compression line. Such a result violates for sure the second law of thermodynamics since the heat losses are negligible during the compressor operation (Aritomi et al., 2011). This fact clearly indicates the strong influence of the measurement uncertainties on the reliability of the fluid properties determined from the measured data.
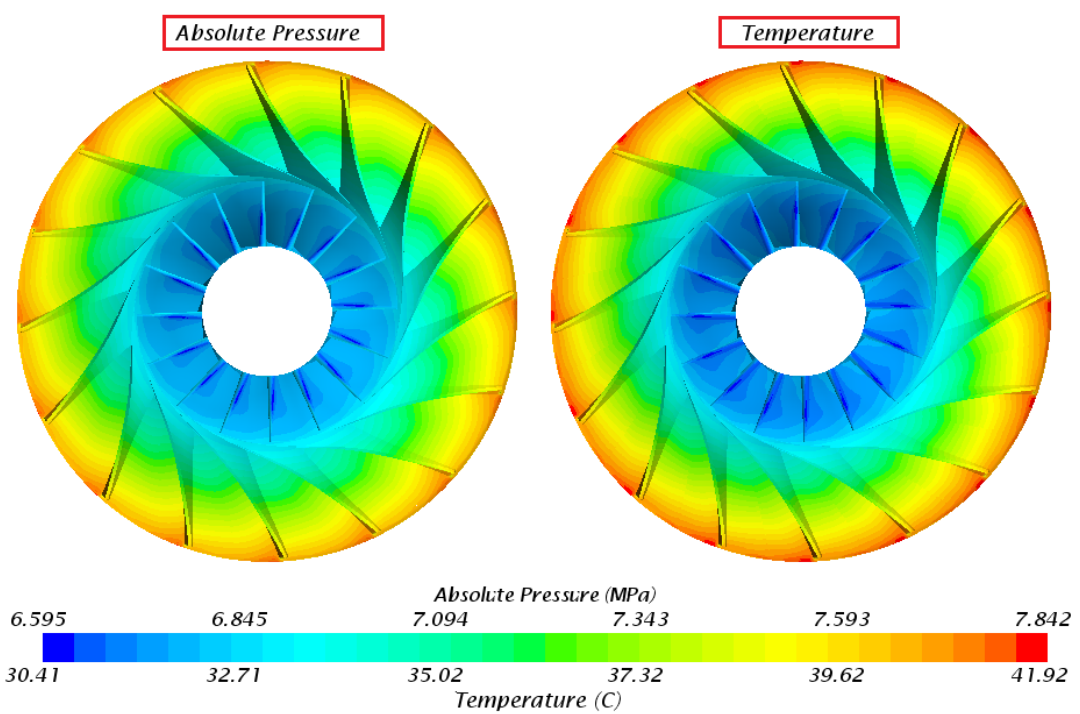

Fig 8: Pressure contour (left) and temperature contour (right) on the impeller blade and hub surface at the on-design operating conditions of the TiTech compressor.

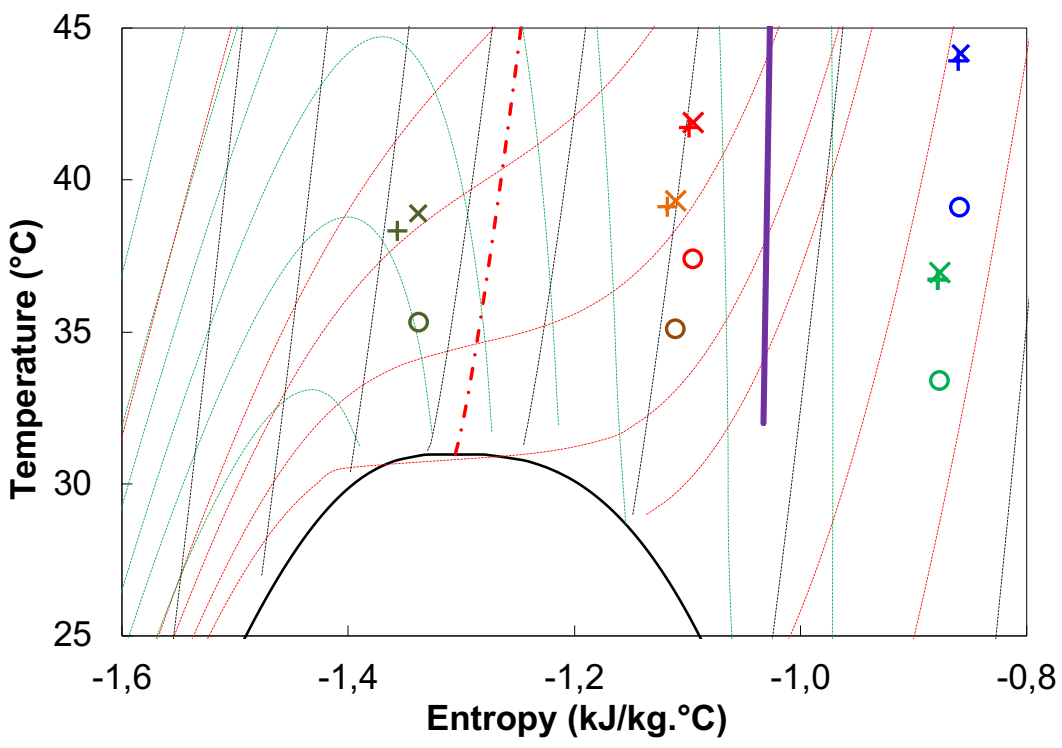

Fig 9: Distribution of TiTech experimental data used for the simulations and CFD results of the corresponding conditions.

Table 5: Comparison of experimental data and CFD results on the TiTech compressor. First line represents CFD results at the on-design conditions. 


\begin{tabular}{|c|c|c|c|c|c|c|c|c|c|}
\hline \multicolumn{4}{|c|}{ Compressor input } & \multicolumn{4}{|c|}{ Compressor output } & \multicolumn{2}{|c|}{ Errors } \\
\hline \multirow{2}{*}{$N(\mathrm{rpm})$} & \multirow{2}{*}{$\dot{\mathrm{m}}(\mathrm{kg} / \mathrm{s})$} & \multirow{2}{*}{$\mathrm{T}_{\text {in }}\left({ }^{\circ} \mathrm{C}\right)$} & \multirow{2}{*}{$P_{\text {in }}(\mathrm{MPa})$} & \multicolumn{2}{|c|}{$\mathrm{T}_{\text {out }}\left({ }^{\circ} \mathrm{C}\right)$} & \multicolumn{2}{|c|}{$\mathrm{P}_{\text {out }}(\mathrm{MPa})$} & \multirow{2}{*}{$\begin{array}{l}\Delta \mathrm{T}_{\text {out }} \\
\left({ }^{\circ} \mathrm{C}\right)\end{array}$} & \multirow{2}{*}{$\begin{array}{c}\Delta \mathrm{P}_{\text {out }} \\
(\mathrm{MPa})\end{array}$} \\
\hline & & & & CFD & EXP & CFD & EXP & & \\
\hline 24000 & 2.00 & 32.00 & 6.800 & 45.09 & $\mathrm{n} / \mathrm{a}$ & 8.074 & $\mathrm{n} / \mathrm{a}$ & $\mathrm{n} / \mathrm{a}$ & $\mathrm{n} / \mathrm{a}$ \\
\hline 13100 & 1.15 & 33.44 & 5.330 & 36.96 & 36.74 & 5.569 & 5.580 & 0.22 & -0.011 \\
\hline 15510 & 1.34 & 39.14 & 5.500 & 44.17 & 43.94 & 5.842 & 5.860 & 0.23 & -0.018 \\
\hline 13104 & 3.74 & 35.20 & 7.624 & 39.32 & 39.20 & 8.120 & 8.145 & 0.12 & -0.025 \\
\hline 13710 & 3.72 & 37.40 & 7.792 & 41.91 & 41.74 & 8.332 & 8.340 & 0.17 & -0.008 \\
\hline 12000 & 4.01 & 35.35 & 8.271 & 38.91 & 38.34 & 9.117 & 9.130 & 0.57 & -0.013 \\
\hline
\end{tabular}

The measurement reliability further demonstrates its importance when evaluating the compressor performance. Table 6 reports, for the two latter cases in Table 5, the enthalpy rises within the compressor derived from the measured temperatures and pressures at the compressor inlet and outlet, in comparison with those given by the CFD simulations. Taking into account the sensor uncertainties, the enthalpy rises vary in a very large range, covering negative to positive values. The absolute values of lower and upper bounds of these ranges are much larger than the enthalpy rises determined from CFD results.

Table 6: Enthalpy rises derived from the measured temperature and pressure and those given by the CFD simulations.

\begin{tabular}{|l|c|c|c|c|c|c|}
\hline \multirow{2}{*}{} & \multicolumn{2}{|c|}{ Input data } & \multicolumn{2}{c|}{ Output data } & \multicolumn{2}{c|}{ Performance } \\
\cline { 2 - 7 } & $\mathrm{T}_{\text {in }}\left({ }^{\circ} \mathrm{C}\right)$ & $\mathrm{P}_{\text {in }}(\mathrm{MPa})$ & $\mathrm{T}_{\text {out }}\left({ }^{\circ} \mathrm{C}\right)$ & $\mathrm{P}_{\text {out }}(\mathrm{MPa})$ & $\Delta \mathrm{H}_{\mathrm{t}}(\mathrm{kJ} / \mathrm{kg})$ & $\eta(\%)$ \\
\hline EXP & $37.40 \pm 0.5$ & $7.792 \pm 0.02$ & $41.74 \pm 0.5$ & $8.332 \pm 0.02$ & {$[-6.4 ; 8.3]$} & $\mathrm{n} / \mathrm{a}$ \\
\hline CFD & 37.40 & 7.792 & 41.91 & 8.332 & 1.20 & 88.2 \\
\hline EXP & $35.35 \pm 0.5$ & $8.271 \pm 0.02$ & $38.34 \pm 0.5$ & $9.130 \pm 0.02$ & {$[-21.6 ; 9.9]$} & $\mathrm{n} / \mathrm{a}$ \\
\hline CFD & 35.35 & 8.271 & 38.91 & 9.117 & 1.78 & 86.1 \\
\hline
\end{tabular}

The reported calculation emphasizes the strong impact of the measurement uncertainty on the enthalpy rise derived from the measured temperature and pressure. The same behavior is observed for other thermodynamic properties such as density and entropy (cf. Fig 9). This is a result of the real gas behavior of the working fluid which features dramatic properties variation in the near critical region. Higher accuracy sensors (i.e. thermocouple and barometer) cannot overcome this difficulty, as demonstrated in (Wahl, 2009). This latter work shows that a complementary measurement of the fluid density is essential to derive reliable performance of the sc- $-\mathrm{CO}_{2}$ compressor.

\subsubsection{Simulation results on a full scale compressor}

Initial simulations were performed at the on-design operating conditions of the compressor (c.f. Table 7) using the tabular Span-Wagner equation of state but this could not lead to a converged solution. Although efforts were made to adapt some parameters of the solvers that control the numerical methods, no improvement was achieved. This was likely due to the appearance of twophase zone in the fluid flow. Such a situation would lead to the discontinuity of fluid properties, having implications on the solution stability during the iterative process. This could have been overcome using the modified equation of state but such a process would not have coped with the physical unsoundness of the achieved solution. Therefore, the operating conditions of the compressor were also modified to ensure the achievement of a physically meaningful solution, i.e. a converged solution without the appearance of two-phase region. It was at first privileged to operate the compressor at the on-design conditions of inlet temperature and pressure, i.e. in the 
liquid region and close to the critical point. Hence, the mass flowrate and shaft speed remained the two free parameters that could be changed to reach a converged solution. This will for sure not comply with compressor specifications for an SMR application, but will allow investigating the real gas aspects of the compression, which is the main point of this study. Let's first confine our interest at the inlet conditions that features relatively zero incidence angle.

A set of these two parameters has been found to meet the above mentioned objective without degrading too much the compressor performance, in particular the pressure ratio. It is reported in Table 7 together with the simulation results, in comparison with the on-design conditions. A pressure ratio of 2.19 has been obtained which is viewed to be sufficiently high to characterize the compression in a full scale compressor. The surprisingly high efficiency of $92.6 \%$, compared to the on-design efficiency, deserves some discussions. First, the parasitic losses have not been taken into account in this efficiency since the CFD modeling deals only with the main flow across the compressor. Note that parasitic losses such as disk friction loss, tip clearance loss, and recirculation loss are originating from minor flows leaking away from the main flow. Accounting for these losses would further decrease the compressor performance. Second, the compression of a real working fluid as sc- $\mathrm{CO}_{2}$ in a full scale compressor has not been fully experienced at this stage. Hence, the widely accepted efficiency of ideal gas compressors should be viewed as a qualitative indicator for comparison. Finally, this potentially high efficiency seems to be somehow in line with that of an axial compressor previously sized by Muto et al. (2003).

Table 7: Compressor performances provided by the design tool at the on-design conditions and those resulted from the CFD simulation for a given case study.

\begin{tabular}{|c|c|c|c|c|c|c|c|c|c|}
\hline Case study & $\underset{(\mathrm{rpm})}{\mathrm{N}}$ & $\begin{array}{c}\mathrm{m} \\
(\mathrm{kg} / \mathrm{s})\end{array}$ & $\begin{array}{l}\mathrm{T}_{\mathrm{t} \text {,in }} \\
\left({ }^{\circ} \mathrm{C}\right)\end{array}$ & $\begin{array}{c}\mathrm{P}_{\mathrm{t} \text {,in }} \\
(\mathrm{MPa})\end{array}$ & $\begin{array}{c}V_{\text {in }} \\
(\mathrm{m} / \mathrm{s})\end{array}$ & $\begin{array}{l}\mathrm{T}_{\text {t,out }} \\
\left({ }^{\circ} \mathrm{C}\right)\end{array}$ & $\begin{array}{l}P_{t, \text { out }} \\
(\mathrm{MPa})\end{array}$ & PR & $\eta$ \\
\hline On-design & 7000 & 2324 & \multirow{2}{*}{27.0} & \multirow{2}{*}{8.40} & 62.8 & 43.4 & 20.00 & 2.38 & $89.0 \%$ \\
\hline ¡1 & 5987 & 1482 & & & 39.6 & 41.1 & 18.39 & 2.19 & $92.6 \%$ \\
\hline
\end{tabular}

The contours of temperature and pressure are shown in Fig 10 and Fig 11. Overall, there is no discontinuity in the evolution of these parameters, illustrating the achievement of a physically meaningful solution. In addition, these figures disclose the dramatic decrease in the temperature and pressure in the region near the impeller leading edge where the flow acceleration takes place. Minimum temperature and pressure of $22.1{ }^{\circ} \mathrm{C}$ and $6.05 \mathrm{MPa}$ have been respectively found, in comparison with the total temperature and total pressure of $27.0{ }^{\circ} \mathrm{C}$ and $8.40 \mathrm{MPa}$ at the compressor inlet. This minimum pressure is slightly above the $6.02 \mathrm{MPa}$ saturation pressure at the achieved minimum temperature, implying a very high potentiality of entering the saturation dome.

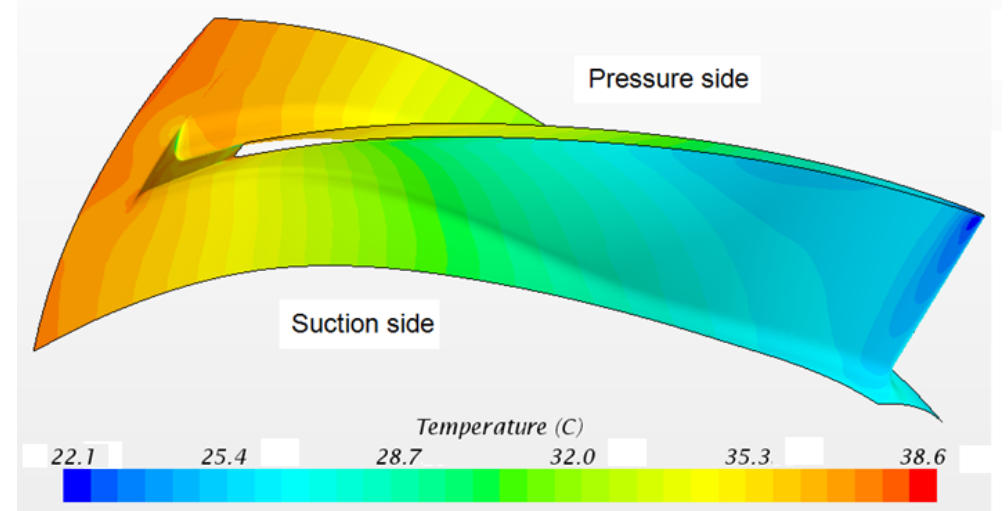

Fig 10: Temperature contour on the impeller blade and hub surfaces of the full scale sc- $\mathrm{CO}_{2}$ compressor. 


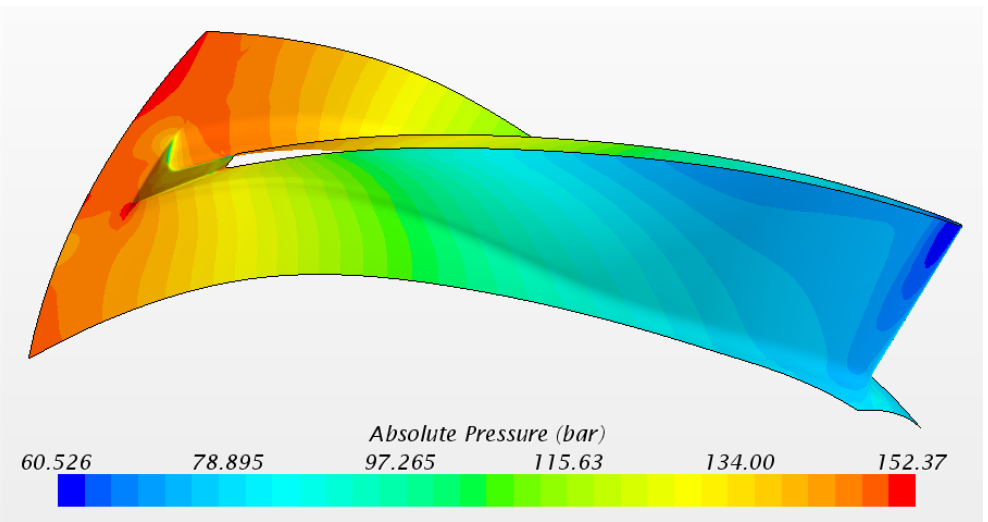

Fig 11: Pressure contour on the impeller blade and hub surfaces of the full scale sc- $\mathrm{CO}_{2}$ compressor.

In order to gain a more quantitative understanding of these decreases, a simple analytical calculation has been performed. It consists in determining the evolution of the static temperature and static pressure during the acceleration of flow under the isentropic condition. Both ideal gas and $\mathrm{sc}-\mathrm{CO}_{2}$ have been considered at a total temperature and a total pressure of $27{ }^{\circ} \mathrm{C}$ and 8.40 $\mathrm{MPa}$, respectively. A comparison between both thermodynamic models is reported in Fig 12. Also plotted therein is the saturation pressure of $\mathrm{CO}_{2}$ derived from the corresponding temperature. These plots clearly demonstrate the dramatic decrease in temperature and pressure in the real gas flow. Additionally, the $\mathrm{CO}_{2}$ pressure is just slightly above the saturation pressure at high velocity, around $80 \mathrm{~m} / \mathrm{s}$. It suggests that a further increase in the flow speed would let the fluid enter the two-phase region.
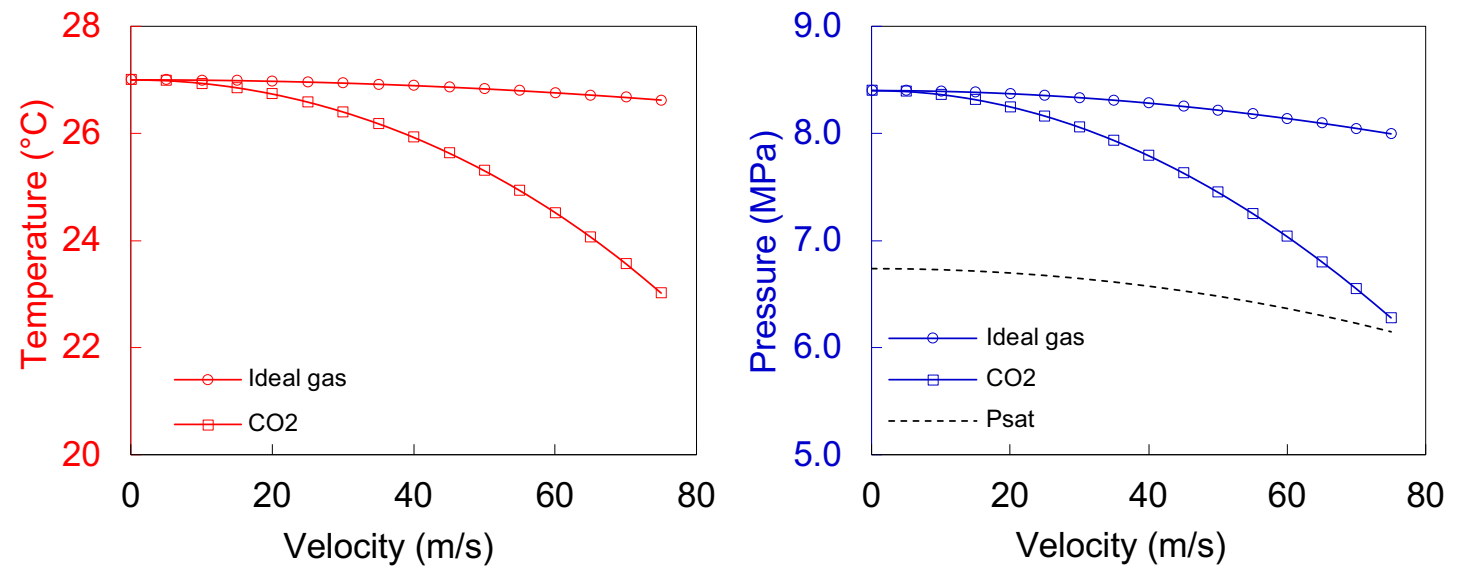

Fig 12: Temperature and pressure decreases in the $\mathrm{sc}-\mathrm{CO}_{2}$ and ideal gas flow acceleration.

The high potential risk of crossing the saturation dome supports the aforementioned fact that there was no possibility to achieve a physical solution by increasing the mass flowrate and shaft speed to approach the on-design operating conditions. Compressor operation at an inlet temperature of $35^{\circ} \mathrm{C}$ is addressed in the following simulations. Note that such a temperature was recommended in the literature in view of accommodating the cycle to the world-wide heat sinks possibilities (Dostal, 2004). Two case studies in the supercritical condition, namely i2 and i3, are considered.

Table 8: Comparison of compressor operating conditions for three case studies.

\begin{tabular}{|c|c|c|c|c|c|}
\hline Case study & $\mathrm{N}(\mathrm{rpm})$ & $\dot{m}(\mathrm{~kg} / \mathrm{s})$ & $\mathrm{T}_{\mathrm{t}, \mathrm{in}}\left({ }^{\circ} \mathrm{C}\right)$ & $\mathrm{P}_{\mathrm{t}, \text { in }}(\mathrm{MPa})$ & $\mathrm{V}_{\text {in }}(\mathrm{m} / \mathrm{s})$ \\
\cline { 1 - 3 } $\mathrm{i} 1$ & \multirow{3}{*}{5987} & 1482 & 27.0 & 8.40 & \multirow{2}{*}{39.6} \\
\cline { 1 - 3 } $\mathrm{i} 2$ & 1144 & 35.0 & 8.40 & \multirow{2}{*}{$\mathrm{i}$} \\
\cline { 1 - 3 } $\mathrm{i} 3$ & & 1293 & 35.0 & 9.10 & \\
\cline { 4 - 5 } & & & &
\end{tabular}




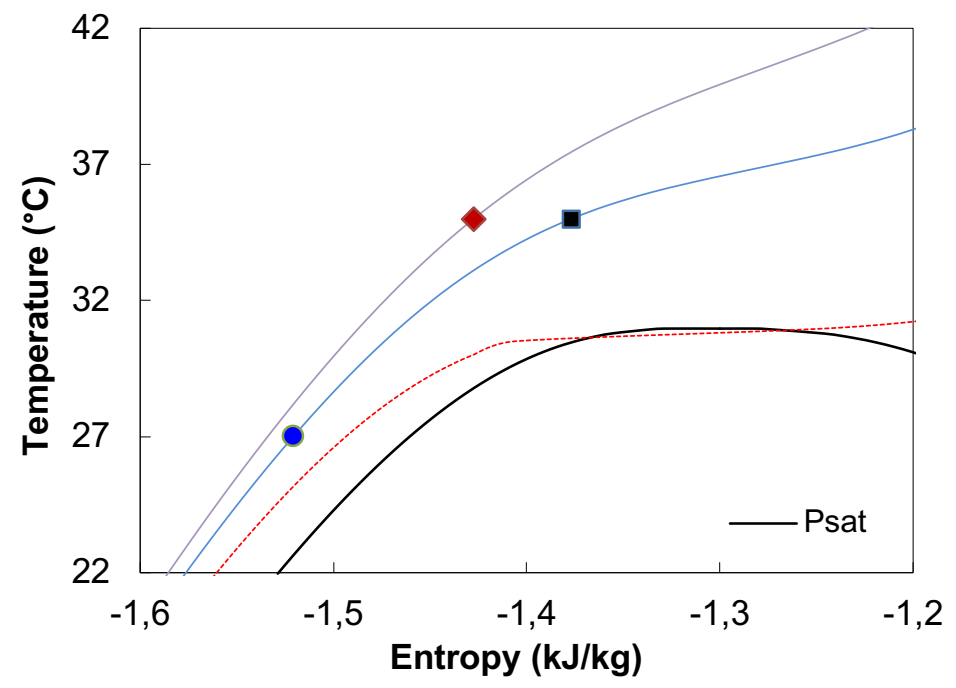

Fig 13: Compressor inlet conditions for three cases i1, i2 and i3.

The inlet pressure is maintained at $8.4 \mathrm{MPa}$ in case i2 (which is close to the optimal value for the highest cycle efficiency) and increased to $9.1 \mathrm{MPa}$ in case i3. This latter is somewhat a choice that may favor stable operation of the compressor in a region of smoother change of fluid properties. The compressor shaft speed is the same in all three cases but the mass flowrates are adjusted to have the equivalent velocity inlet in order to keep the operation of the compressor at the same optimal incidence angle.

Simulations have been carried out on these two additional case studies. As the modified equation state is utilized, the converged solution is always assured even if there is the appearance of a biphasic region inside the compressor. Thanks to this final solution, one can track the state change of the working fluid and then assess the physical soundness of the solution (i.e. by verifying the lack of two-phase flow condition). This idea is demonstrated in Fig 14 where the colors indicate different fluid phases with the convention shown in the color bar. In the first case, the compressor inlet is in the liquid region, the working fluid is compressed to the supercritical region. The liquid-supercritical phase transition takes place smoothly inside the compressor. In the second case, although the inlet conditions are shifted into the supercritical region (with higher inlet temperature), there is a large biphasic region (the blue region) that develops near the leading edge of the impeller blades. With the increase in the inlet pressure from 8.4 to $9.1 \mathrm{MPa}$, the two-phase zone no longer exists within the compressor. In this case, the compressor operates in the supercritical region, except a small fraction of liquid phase near the leading edge.

These simulation results suggest that compressor operating at a supercritical temperature does not necessarily provide a larger margin to the saturation dome than that at subcritical temperature. Indeed, it is the inlet pressure which should also be chosen in accommodation with the inlet temperature. The commonly considered inlet conditions for optimal cycle efficiency (i.e. case i2) shows high risks of two-phase flow appearance inside the compressor. This situation can be prevented through two possibilities which consist in modifying either the inlet pressure or the inlet temperature. The former leads to degradation in the thermal efficiency of the sc- $\mathrm{CO}_{2}$ cycle whereas the latter provide higher cycle performance (see Floyd et al., 2011). The initial choice of the inlet condition in the subcritical liquid region, which was actually made to optimize the cycle performance, interestingly shows an additional benefit in terms of preventing the appearance of the two-phase condition inside the compressor. 


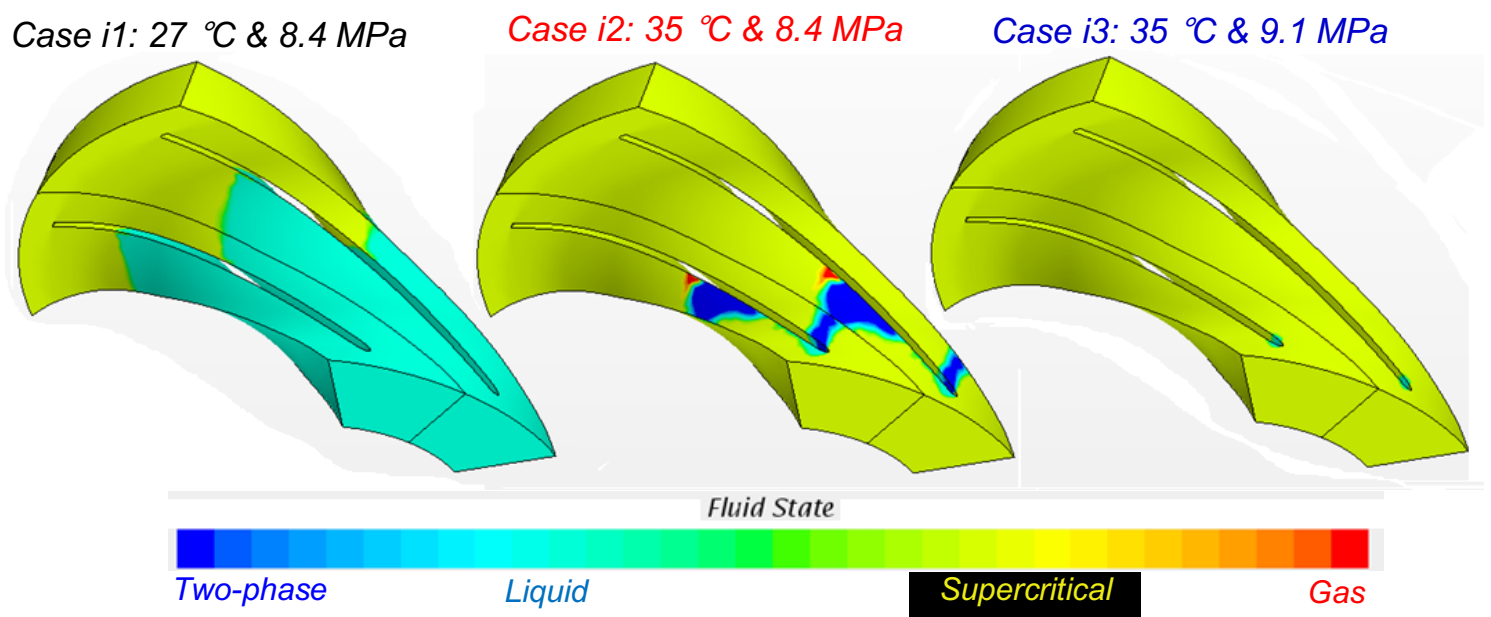

Fig 14: State change of the fluid inside the sc- $\mathrm{CO}_{2}$ compressor for three case studies. The modeled fluid domain is duplicated for the representation purpose.

In the aforementioned case studies, the inlet velocity and the shaft speed have been chosen so that the flow entering the impeller remains close to the optimal incidence angle. In such configuration, the acceleration of the flow past the leading edge is minimal. If this is not the case, higher flow velocity could be expected and thus more potentiality of crossing the saturation dome can be foreseen. To confirm this point, the mass flowrates in cases i 1 and i3 have been adjusted to increase the inlet velocity by $15 \%$ while the other conditions are maintained. The simulation results of these two new cases, namely $11^{*}$ and $3^{*}$, are shown in Fig 15 . For each case, a small two-phase area is marked on the pressure side of the impeller entrance region. The case $13^{*}$, in comparison to its original case $\mathrm{i} 3$, shows a larger liquid region which develops from the leading edge to the suction side of the next blade. The case $11^{*}$ features a second small two-phase area in the suction side of the impeller blade, which is in line with the depressurization and the temperature drop in this place.

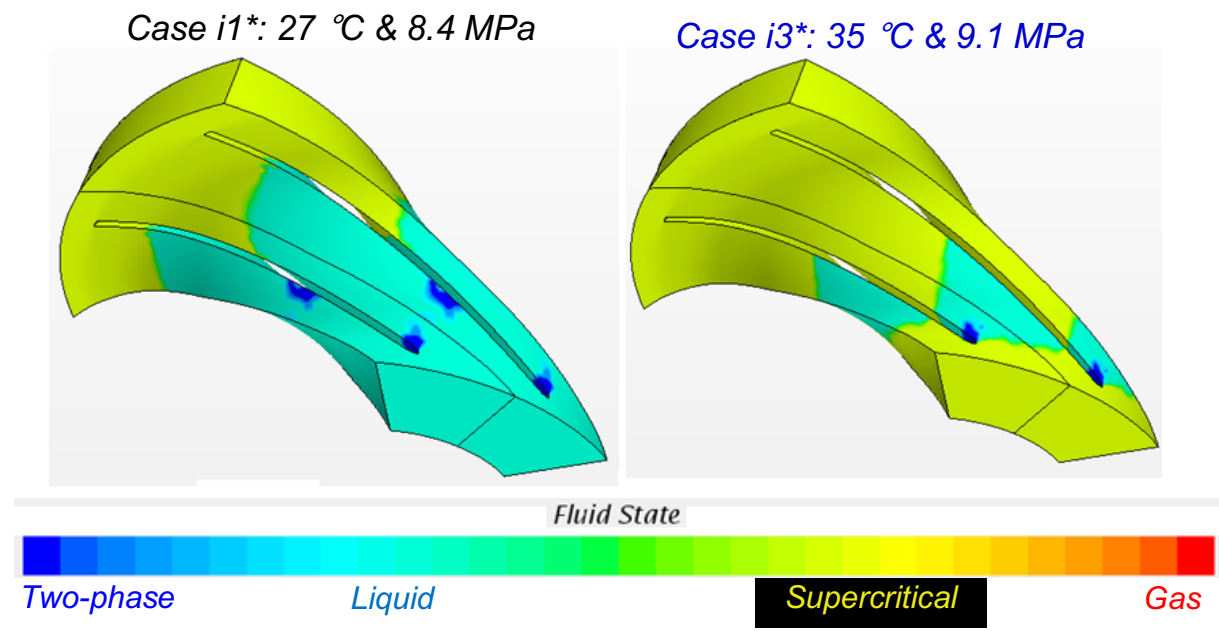

Fig 15: Sate change of the fluid inside the $\mathrm{sc}-\mathrm{CO}_{2}$ compressor for two modified case studies. The modeled fluid domain is duplicated for the representation purpose.

These complementary simulation results show that the previous recommendation in the choice of the inlet temperature and pressure is not sufficient to assure a margin to the saturation dome. A small velocity inlet, together with a low rotation speed, is additionally required to keep the flow acceleration under a certain limitation. There is actually no quantitative indication that can generally be given at this stage due to the fact that its relevance would be limited to the considered compressor geometry and other factors to be defined. Among these factors, the range of off-design operating conditions should be paramount. For example, a larger deviation of the flow entering the 
impeller with respect to the optimal incidence angle would lead to more significant flow acceleration and thus would require a smaller inlet relative velocity at the on-design conditions. Design of the leading edge should also impact the flow acceleration. To conclude, the prevention of the twophase flow in the sc- $\mathrm{CO}_{2}$ compressor requires coping with the effect of flow acceleration near the impeller leading edge at both on- and off-design operating conditions. Clearly, these constraints will be relaxed if the appearance of a two-phase region inside the compressor is proven to not yield marked performance degradation, neither severe detrimental mechanics effect, which could be in line with the aforementioned observations at SNL.

\section{Performance characteristics of the $\mathrm{sc}-\mathrm{CO}_{2}$ compressor}

\subsection{Revision of the previous performance maps approaches}

Three performance maps approaches are considered in this revision and their dimensionless parameters are recapitulated in Table 9. The pressure ratio and the efficiency are already dimensionless parameter and thus are not shown in this table. Those of the conventional ideal gas approach (IG) can, as previously mentioned, be derived through the dimensional analysis (cf. Roberts and Sjolander, 2005). Indeed, the reported dimensionless enthalpy rise is not commonly used to define the performance of an ideal gas compressor. For such a purpose, the pressure ratio, as well as the efficiency, is employed. Nevertheless, these three parameters will be examined in the following, even if two of them are sufficient to determine the compressor performance. Additionally, for the sake of convenience, the corrected parameters, which are formulated from these dimensionless parameters at the actual conditions (cf.

Table 10), are used to establish the performance maps at the considered reference conditions.

If the gas does not follow the ideal gas law and its departure from the ideal gas can be characterized through the compressibility factor, more general parameters, as given in (Kidnay et al., 2011), can be used. Such a case is hereafter named as ideal gas approach with compressibility correction (IGZ). It is important to highlight that these two correction approaches can only be valid within the assumption of constant specific heat ratio. When this assumption is not satisfactory, the critical conditions, instead of the total conditions, could be referred to, as is the case of the BNI approach. The temperature and pressure at the critical conditions in these dimensionless parameters can be determined through those at the total conditions as follows:

$$
Z_{c r} T_{c r}=Z_{t} T_{t}\left(\frac{1+\gamma}{2}\right)^{-1} \quad \text { (Eq. 1) } \quad P_{c r}=P_{t}\left(\frac{1+\gamma}{2}\right)^{-\frac{\gamma}{\gamma-1}}
$$

Table 9: Dimensionless parameters of different approaches for establishing the turbomachinery performance map.

\begin{tabular}{|c|c|c|c|}
\hline & IG & IGZ & BNI \\
\hline$\dot{m}_{a d}$ & $\frac{\dot{m} \sqrt{r r T_{t}}}{\gamma P_{t}}$ & $\frac{\dot{m} \sqrt{r r Z_{t} T_{t}}}{\gamma P_{t}}$ & $\frac{\dot{m} \sqrt{r Z_{c r} T_{c r}}}{\gamma P_{c r}}$ \\
\hline$N_{a d}$ & $\frac{N}{\sqrt{r r T}}$ & $\frac{N}{\sqrt{r Z_{t} T_{t}}}$ & $\frac{N}{\sqrt{r Z_{c r} T_{c r}}}$ \\
\hline$\Delta H_{a d}$ & $\frac{\Delta H_{t}}{\gamma r T_{t}}$ & $\frac{\Delta H_{t}}{r Z_{t} T_{t}}$ & $\frac{\Delta H_{t}}{\gamma r Z_{c r} T_{c r}}$ \\
\hline
\end{tabular}


Table 10: Corrected parameters of different approaches for establishing the turbomachinery performance map. Subscripts $a$ and $r e f$ denote the actual and reference conditions, respectively.

\begin{tabular}{|c|c|c|c|}
\hline$\dot{m}_{c}$ & $\left(\dot{m} \frac{\sqrt{r r T_{t}}}{\gamma P_{t}}\right)_{a}\left(\frac{\gamma P_{t}}{\sqrt{r r T_{t}}}\right)_{r e f}$ & $\left(\dot{m} \frac{\sqrt{r r Z_{t} T_{t}}}{\gamma P_{t}}\right)_{a}\left(\frac{\gamma P_{t}}{\sqrt{r r Z_{t} T_{t}}}\right)_{r e f}$ & $\left(\dot{m} \frac{\sqrt{r r Z_{c r} T_{c r}}}{\gamma P_{c r}}\right)_{a}\left(\frac{\gamma P_{c r}}{\sqrt{r r Z_{c r} T_{c r}}}\right)_{r e f}$ \\
\hline$N_{c}$ & $\left(N \frac{1}{\sqrt{r r T_{t}}}\right)_{a}\left(\sqrt{r r T_{t}}\right)_{r e f}$ & $\left(N \frac{1}{\sqrt{r r Z_{t} T_{t}}}\right)_{a}\left(\sqrt{r r Z_{t} T_{t}}\right)_{r e f}$ & $\left(N \frac{1}{\sqrt{r r Z_{c r} T_{c r}}}\right)_{a}\left(\sqrt{r r Z_{c r} T_{c r}}\right)_{r e f}$ \\
\hline$\Delta H_{c}$ & $\Delta H_{t}\left(\frac{1}{\gamma r T_{t}}\right)_{a}(\gamma r T)_{r e f}$ & $\left(\Delta H_{t} \frac{1}{r r Z_{t} T_{t}}\right)_{a}\left(r r Z_{t} T_{t}\right)_{r e f}$ & $\left(\Delta H_{t} \frac{1}{r r Z_{c r} T_{c r}}\right)_{a}\left(r r Z_{c r} T_{c r}\right)_{r e f}$ \\
\hline
\end{tabular}

In brief, the non-ideal gas behavior was previously taken into account through the compressibility factor and the critical condition. The former provides a correction for the state variables, specifically the density, to cope with the change of inlet temperature and pressure while the latter is seen to allow overcoming the variation of specific heat ratio. In the following subsections, we will examine step by step the effect of each refinement in the case of a sc- $\mathrm{CO}_{2}$ compressor.

\subsubsection{Effects of the compressibility correction}

Let's first consider some inlet conditions which satisfy the assumption of constant specific heat ratio. As such, the contribution of the compressibility factor can be made clear by comparing the compressor performance at different inlet conditions following each approach. For this purpose, CFD simulations on the full scale sc- $\mathrm{CO}_{2}$ compressor have been carried out, providing results to calculate the performance parameters (i.e. corrected enthalpy rise, pressure ratio and efficiency). The inlet conditions have been chosen in the $27-40{ }^{\circ} \mathrm{C}$ temperature range and $9.0-12.3 \mathrm{MPa}$ pressure range so that the variation of the compressibility factor is significant. The mass flowrate and shaft speed corresponding to each point have been chosen to yield unique corrected mass flowrate and corrected speed, being set at $1150 \mathrm{~kg} / \mathrm{s}$ and $4500 \mathrm{rpm}$. The compressor performance following the IG and IGZ approaches are recapitulated in Table 11, together with their maximum discrepancies. These are given in relative values for the corrected enthalpy rise and in in absolute values for pressure ratio and efficiency.

Table 11: Performance comparison between the IG and IGZ correction approaches at corrected mass flowrate and speed of $1150 \mathrm{~kg} / \mathrm{s}$ and $4500 \mathrm{rpm}$ with respect to the reference conditions of $27^{\circ} \mathrm{C}$ and $9.00 \mathrm{MPa}$.

\begin{tabular}{|c|c|c|c|c|c|c|c|c|c|}
\hline \multirow[b]{2}{*}{$\begin{array}{l}T_{\mathrm{t}, \mathrm{in}} \\
\left({ }^{\circ} \mathrm{C}\right)\end{array}$} & \multirow[b]{2}{*}{$\begin{array}{c}P_{\mathrm{t}, \mathrm{in}} \\
(\mathrm{MPa})\end{array}$} & \multirow[b]{2}{*}{$Z_{t, i n}$} & \multirow[b]{2}{*}{$\gamma$} & \multicolumn{3}{|c|}{ IG approach } & \multicolumn{3}{|c|}{ IGZ approach } \\
\hline & & & & $\begin{array}{c}\Delta \mathrm{H}_{\mathrm{c}} \\
(\mathrm{kJ} / \mathrm{kg})\end{array}$ & PR & $\eta(\%)$ & $\begin{array}{c}\Delta \mathrm{H}_{\mathrm{c}} \\
(\mathrm{kJ} / \mathrm{kg})\end{array}$ & PR & $\eta(\%)$ \\
\hline 27.0 & 9.00 & 0.204 & 3.46 & 6.82 & 1.558 & 92.67 & 6.82 & 1.558 & 92.67 \\
\hline 30.0 & 9.84 & 0.224 & 3.46 & 6.51 & 1.485 & 92.62 & 6.83 & 1.559 & 92.63 \\
\hline 35.0 & 11.12 & 0.256 & 3.46 & 6.04 & 1.390 & 91.89 & 6.85 & 1.563 & 92.55 \\
\hline 40.0 & 12.29 & 0.286 & 3.46 & 5.60 & 1.317 & 90.22 & 6.87 & 1.568 & 92.66 \\
\hline \multicolumn{4}{|c|}{ Maximum discrepancy } & $18 \%$ & 0.241 & 2.45 & $0.7 \%$ & 0.01 & 0.12 \\
\hline
\end{tabular}


Table 11 shows that a given couple of corrected speed and corrected mass flowrate following the IG approach does not result in the same pressure ratio and corrected enthalpy rise at different inlet temperatures and pressures. The discrepancy in the efficiency is less pronounced. However, since the outlet pressure predicted by the performance maps encounters a significant error, any accurate prediction in the efficiency becomes useless. Taking into account the compressibility factor, the IGZ approach provides better consistency for the corrected enthalpy rise and pressure ratio. Among the four case studies, the maximum discrepancy found on the enthalpy rise parameter is less than $1 \%$ and that for the compressor outlet pressure (calculated through the pressure ratio discrepancy and the inlet pressure) is below $0.12 \mathrm{MPa}$. This comparison suggests that the IGZ approach can be reliably used to represent the similarity conditions as long as the specific heat ratio is maintained constant.

\subsubsection{Effects of the critical conditions}

In order to examine the effects of this later, CFD simulations have been performed at several inlet conditions which yield different heat specific ratios, as summarized in Table 12. Both IGZ and $\mathrm{BNI}$ approaches provide very good consistency for the efficiency (maximum discrepancy of 0.32 point) and the corrected enthalpy rise (maximum discrepancy of $2.1 \%$ ) with respect to the specific heat ratio variation. Regarding the pressure ratio, that provided by IGZ approach now varies in a such noticeably wide range that the maximum discrepancy in the outlet pressure can be up to 2 $\mathrm{MPa}$. This latter is only $0.44 \mathrm{MPa}$ for the BNI approach, showing a significant improvement compared to the IGZ approach. Nevertheless, it is important to remind that the performance of the compressor at a given state can be fully defined using two among three parameters: pressure ratio, enthalpy rise, and efficiency. As such, the enthalpy rise and efficiency can be used to reliably represent the similarity conditions within the variation of the specific heat ratio in the range mentioned in Table 12.

Although the discrepancies of the corrected enthalpy rise and of the efficiency reported in Table 12 still remains in a small range, larger errors could be expected with more significant variation of the specific heat ratio. Note that this latter changes from a value close to unity in the gas phase to very high values in the pseudocritical line, even approaches infinite in the vicinity of the critical point. This issue has been considered through another comparison between the IGZ and BNI approach, as shown in Table 13. Face to a significant change of the specific heat ratio, from 2.41 to 24.5 , both approaches can no longer retain their consistent representation for the sc$\mathrm{CO}_{2}$ compressor performance. Regarding the corrected enthalpy, the IGZ approach encounters a maximum error of $20 \%$ and is reduced to $12 \%$ for $\mathrm{BNI}$ approach, which is still out of a somewhat accurate range. The efficiency discrepancy is now increased up to around 1 point for the IGZ approach and 2 points for the BNI approach. As such, the reliable prediction of these approaches cannot be assured in the whole range of inlet conditions near the critical point.

Table 12: First performance comparison between the IGZ and BNI correction approaches at the corrected mass flowrate and speed of $870 \mathrm{~kg} / \mathrm{s}$ and $4500 \mathrm{rpm}$ with respect to the reference conditions of $40^{\circ} \mathrm{C}$ and $9.60 \mathrm{MPa}$.

\begin{tabular}{|c|c|c|c|c|c|c|c|c|c|}
\hline \multirow[b]{2}{*}{$\begin{array}{l}T_{\mathrm{t}, \mathrm{in}} \\
\left({ }^{\circ} \mathrm{C}\right)\end{array}$} & \multirow[b]{2}{*}{$\begin{array}{c}\mathrm{P}_{\mathrm{t}, \mathrm{in}} \\
(\mathrm{MPa})\end{array}$} & \multirow[b]{2}{*}{$Z_{\mathrm{t}, \mathrm{in}}$} & \multirow[b]{2}{*}{$\gamma$} & \multicolumn{3}{|c|}{ IGZ approach } & \multicolumn{3}{|c|}{ BNI approach } \\
\hline & & & & $\begin{array}{c}\Delta \mathrm{Hc} \\
(\mathrm{kJ} / \mathrm{kg})\end{array}$ & PR & $\eta(\%)$ & $\begin{array}{c}\Delta \mathrm{Hc} \\
(\mathrm{kJ} / \mathrm{kg})\end{array}$ & PR & $\eta(\%)$ \\
\hline 27.0 & 9.00 & 0.204 & 3.46 & 6.79 & 1.200 & 92.54 & 7.12 & 1.366 & 92.41 \\
\hline 30.0 & 9.00 & 0.211 & 3.87 & 6.81 & 1.224 & 92.41 & 7.08 & 1.373 & 92.20 \\
\hline 35.0 & 9.60 & 0.237 & 4.42 & 6.83 & 1.259 & 92.42 & 7.05 & 1.384 & 92.19 \\
\hline 40.0 & 9.60 & 0.274 & 6.75 & 6.93 & 1.412 & 92.22 & 6.93 & 1.412 & 92.25 \\
\hline \multicolumn{4}{|c|}{ Maximum discrepancy } & $2.1 \%$ & 0.212 & 0.32 & $2.7 \%$ & 0.046 & 0.22 \\
\hline
\end{tabular}


Table 13: Second performance comparison between the IGZ and BNI correction approaches at the corrected mass flowrate and speed of $436 \mathrm{~kg} / \mathrm{s}$ and $3087 \mathrm{rpm}$ with respect to the reference conditions of $34{ }^{\circ} \mathrm{C}$ and $7.86 \mathrm{MPa}$.

\begin{tabular}{|c|c|c|c|c|c|c|c|c|c|}
\hline \multirow[b]{2}{*}{$\begin{array}{l}T_{\mathrm{t}, \mathrm{in}} \\
\left({ }^{\circ} \mathrm{C}\right)\end{array}$} & \multirow[b]{2}{*}{$\begin{array}{c}P_{\mathrm{t}, \mathrm{in}} \\
(\mathrm{MPa})\end{array}$} & \multirow[b]{2}{*}{$\mathrm{Z}_{\mathrm{t}, \text { in }}$} & \multirow[b]{2}{*}{$\gamma$} & \multicolumn{3}{|c|}{ IGZ approach } & \multicolumn{3}{|c|}{ BNI approach } \\
\hline & & & & $\begin{array}{c}\Delta \mathrm{H}_{\mathrm{c}} \\
(\mathrm{kJ} / \mathrm{kg})\end{array}$ & PR & $\eta(\%)$ & $\begin{array}{c}\Delta \mathrm{Hc} \\
(\mathrm{kJ} / \mathrm{kg})\end{array}$ & PR & $\eta(\%)$ \\
\hline 34.0 & 6.00 & 0.642 & 2.41 & 4.00 & 1.016 & 91.17 & 3.71 & 1.141 & 90.13 \\
\hline 27.0 & 9.00 & 0.204 & 3.46 & 3.19 & 1.018 & 91.77 & 3.55 & 1.144 & 91.57 \\
\hline 35.0 & 8.05 & 0.303 & 24.5 & 3.27 & 1.135 & 92.14 & 3.28 & 1.171 & 92.22 \\
\hline \multicolumn{4}{|c|}{ Maximum discrepancy } & $20 \%$ & 0.119 & 0.97 & $12 \%$ & 0.030 & 2.09 \\
\hline
\end{tabular}

While the corrected enthalpy rise and efficiency retained in the BNI approach lead to significant errors, it can be noted that the pressure ratio from this approach present a certain improvement. The error in terms of outlet pressure is however still large, around 0.3 MPa, and can be expected to be more significant at higher considered pressure ratios. As such, the contribution of the critical condition is not remarkable for the performance maps representation. In fact, the critical conditions that are used to ensure the similarity conditions in the original work of Glassman (1972), are somewhat unsuited for a flow of $\mathrm{CO}_{2}$ near the critical point. Indeed, as underlined in section 2.2.2, such a high critical velocity of fluid flow is seen to be discarded due to the significant drops in the static temperature and pressure, likely leading to the appearance of the two phase condition (cf. Fig 12). Besides, the use of the heat specific ratio to characterize the fluid during the compression process appears to not be a sound physical choice; the isentropic exponent should have been used instead. Indeed, the fact that this exponent reduces to the specific heat ratio for the ideal gas case does not allow the substitution of the former by the latter in the most general case of working fluid, in particular for sc- $\mathrm{CO}_{2}$ : in contrast to the large variation of the specific heat ratio, the isentropic exponent, as can be seen in the following section, features much smoother change in the region of interest.

\subsection{Development of a sc- $\mathrm{CO}_{2}$ compressor performance maps approach}

Considering the highlighted limitations of the above approaches, there is a comprehensible need in the development of an approach that is able to accommodate the real gas behavior of the working fluid and thus can reliably be used for accurate representation and prediction of the sc$\mathrm{CO}_{2}$ compressor performance. In the following, the key similarities in the turbomachinery problem as well as several thermodynamic formulations for real gases are used to derive adequate dimensionless parameters.

\subsubsection{Derivation of the dimensionless parameters}

In fluid mechanics, various dimensionless numbers which characterize different physical phenomenon related to the flow can be derived on the basis of the scale analysis. For compressible flows without heat transfer such as turbomachinery flows, the important ones that should be retained in defining the similarity conditions are Reynolds, Euler, Mach and Eckert. Their expression, interpretation and typical applications are recapitulated in Table 14. Among others, the effects of the Reynolds number can usually be neglected for flows at sufficiently high Reynolds number (Glassman, 1972). 
Table 14: Common dimensionless number in tuboachinery fluid flows.

\begin{tabular}{|l|l|c|l|}
\hline Number & Expression & Interpretation & \multicolumn{1}{|c|}{ Types of application } \\
\hline Reynolds & $\operatorname{Re}=\frac{\rho V D}{\mu}$ & $\frac{\text { inertial force }}{\text { viscous force }}$ & $\begin{array}{l}\text { All types of fluid mechanics } \\
\text { problems }\end{array}$ \\
\hline Euler & $E u=\frac{P}{\rho V^{2}}$ & $\frac{\text { pressure force }}{\text { Inertial force }}$ & $\begin{array}{l}\text { Flows in which pressure or } \\
\text { pressure difference are of interest }\end{array}$ \\
\hline Mach & $M=\frac{V}{a}$ & $\frac{\text { flow speed }}{\text { speed of sound }}$ & $\begin{array}{l}\text { Flows in which the compressibility } \\
\text { of the fluid is important }\end{array}$ \\
\hline Eckert & $E k=\frac{\Delta H}{V^{2}}$ & $\frac{\text { enthalpy }}{\text { kinetic energy }}$ & $\begin{array}{l}\text { Flows in which the dissipation is } \\
\text { important }\end{array}$ \\
\hline
\end{tabular}

The general expression of the speed of sound reads:

$$
a=\sqrt{n_{s} Z R T}
$$

in which $n_{s}$ denotes the isentropic volume exponent, which characterize the isentropic compression of a real gas as follows:

$$
\left.P v^{n_{s}}\right|_{s}=c t e
$$

This exponent can be linked to the specific heat ratio as follows (Lüdtke, 2004):

$$
n_{S}=-\gamma \frac{v}{P}\left(\frac{\partial P}{\partial v}\right)_{T}
$$

For an ideal gas case, $P=r T / v$, the above equation reduces to:

$$
n_{s}=\gamma
$$

Hence, the exponent $n_{s}$ is a generalized factor that should be used instead of the specific heat ratio to describe the isentropic process of real gases. For $\mathrm{sc}-\mathrm{CO}_{2}$, the variation of the exponent $\mathrm{n}_{\mathrm{s}}$ in the region near the critical point is illustrated in Fig 3-1. In contrast to the specific heat ratio, the isentropic exponent features no dramatic change in the pseudocritical region. 


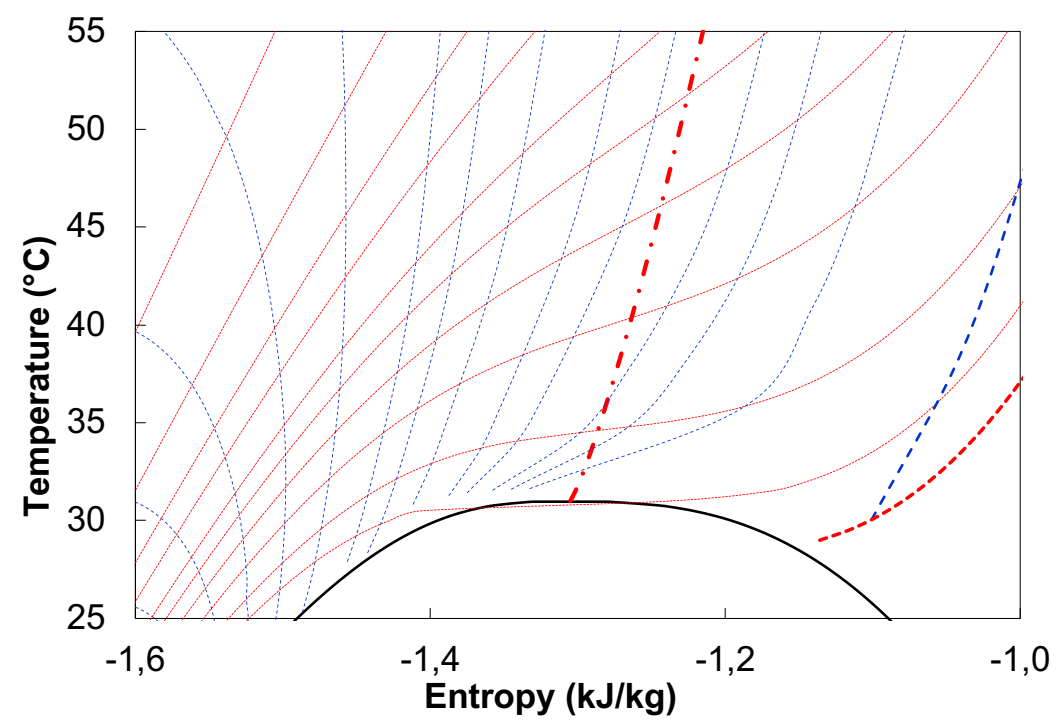

Fig 3-1: Contour of isentropic volume exponent in the T-s diagram, together with different isobaric lines.

The Euler number could be linked to the Mach number, using the equation of state, as follows:

$$
E u=\frac{P}{\rho V^{2}}=\frac{\rho r Z T}{\rho V^{2}}=\frac{n_{s} r Z T}{n_{s} V^{2}}=\frac{1}{n_{s} M^{2}}
$$

Since the Euler number can be related to the Mach number through the isentropic exponent, these latter can be used as an alternative key similarity. As such, the following sets of key similarities should be considered to ensure the similarity conditions: Mach and Eckert numbers, together with the isentropic exponent $n_{s}$.

The dimensionless mass flowrate and dimensionless speed parameters can be derived on the basis of Mach number as follows:

$$
\begin{aligned}
& m_{a d}=\frac{\dot{m} \sqrt{n_{s} r Z_{t} T_{t}}}{n_{s} P_{t}}=M\left(1+\frac{n_{s}-1}{2} M^{2}\right)^{-\frac{n_{s}-1}{2\left(n_{s}-1\right)}} \\
& N_{a d}=\frac{N}{\sqrt{n_{s} r Z_{t} T_{t}}}=k_{v} M\left(1+\frac{n_{s}-1}{2} M^{2}\right)^{-1 / 2}
\end{aligned}
$$

in which $k_{v}=N / V$ is a factor that ensure the similarity of the peripheral speed and $k_{v} M$ can be interpreted elsewhere as the machine Mach number.

For low speed flows, typically $M<0.3$, and thus $M^{2} \ll 1$, the above formulations can be approximated as follows:

$$
m_{a d} \approx M \quad \text { (Eq. 10) } \quad N_{a d} \approx k_{v} M
$$

Note that these parameters do not allow taking into account the similarity of the isentropic exponent and the Eckert number. This latter, nevertheless, should be required to assure the similarity of the dimensionless enthalpy rise:

$$
\Delta H_{a d}=\frac{\Delta H_{t}}{n_{s} r Z_{t} T_{t}}=E k M^{2}\left(1+\frac{n_{s}-1}{2} M^{2}\right)^{-1}
$$




\subsubsection{Preliminary qualification}

In order to qualify this approach, CFD simulations have been carried out at several inlet conditions ranging from the liquid to supercritical phase and featuring large change in the isentropic exponent (marked from c1 to c7 in Fig 2). The mass flowrate and shaft speed have been chosen to yield the same corrected mass flowrate of $1558 \mathrm{~kg} / \mathrm{s}$ and corrected shaft speed of 6300 $\mathrm{rpm}$ at the reference conditions of $27{ }^{\circ} \mathrm{C}$ and $8.4 \mathrm{MPa}$. Performance comparison at these inlet conditions is reported in Fig 3 and Fig 4. It comes out that the corrected enthalpy rise and the efficiency remain relatively constant with respect to the change of inlet conditions. This interestingly suggests that the effect of the Eckert number on the compressor performance is negligible.

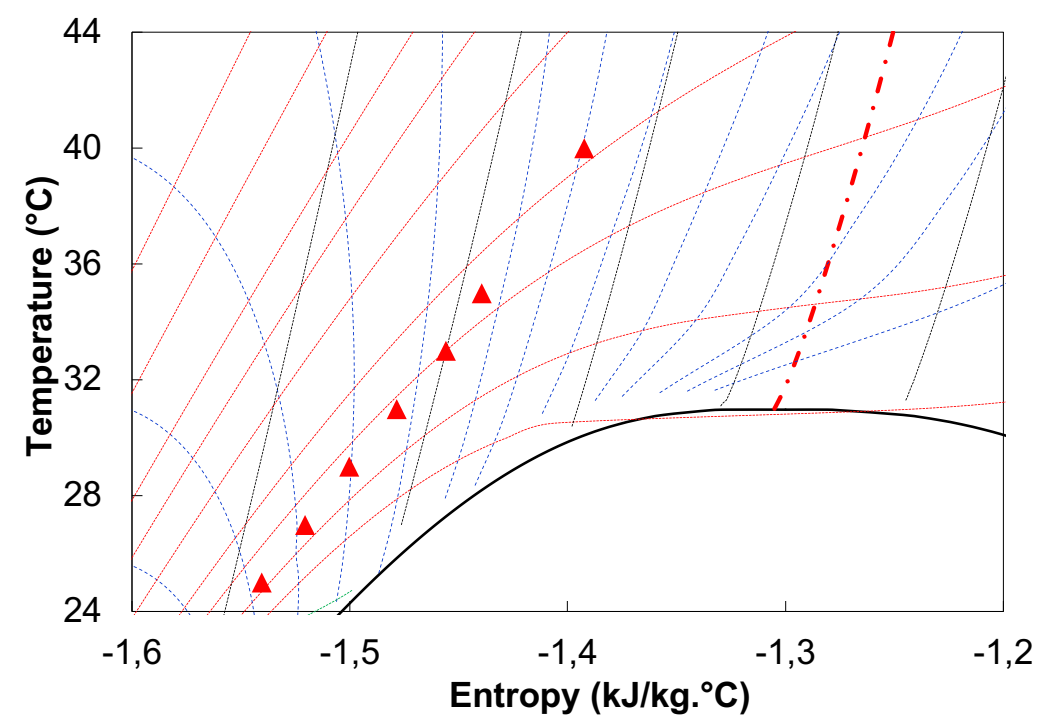

Fig 2: Distribution of compressor inlet conditions used to qualify the performance maps approach for the sc- $\mathrm{CO}_{2}$ compressor.

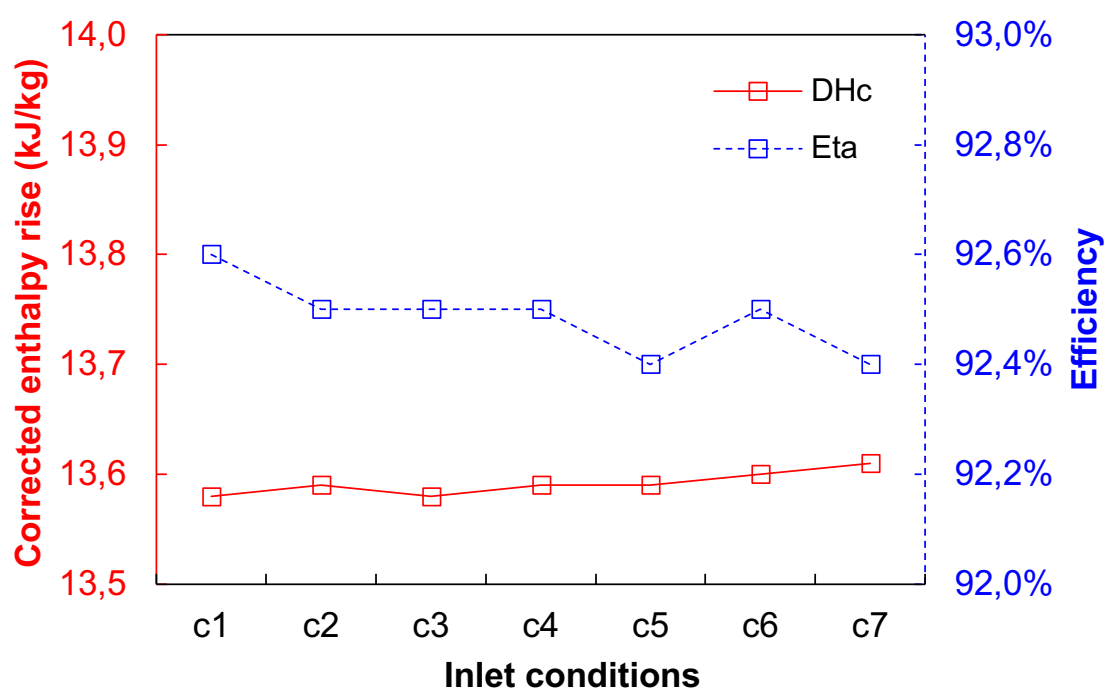

Fig 3: Corrected enthalpy rise (DHc) and efficiency (Eta) at the reference conditions of $27^{\circ} \mathrm{C}$ and 8.40 MPa.

Concerning the pressure ratio, strong variations are noticed and can be explained by the noninclusion of the isentropic exponent (plotted in Fig 4) as part of the key similarities in the derivation of the dimensionless parameters. Even though the similarity of the pressure ratio is not assured, a combination of the enthalpy rise and the efficiency is sufficient to calculate the compressor outlet conditions, as previously mentioned. As such, the above dimensionless parameters provide reliable prediction of the $\mathrm{sc}-\mathrm{CO}_{2}$ compressor performance. 
Nevertheless, using the set of enthalpy rise and efficiency is not as convenient as the conventional set of pressure ratio and efficiency, especially for real gas case. It actually requires the calculation of the temperature and pressure from the entropy and enthalpy. These latter are known to vary non-linearly with respect to the change of temperature and pressure. Hence, an iterative numerical procedure or a look-up table would have to be employed for the mentioned purpose. On contrary, a consistent representation of the pressure ratio would allow for a similar treatment of the sc- $\mathrm{CO}_{2}$ compressor maps to what is currently available for ideal gas compressors in system codes. An additional correction for the pressure ratio is therefore set as the objective of the next subsection.

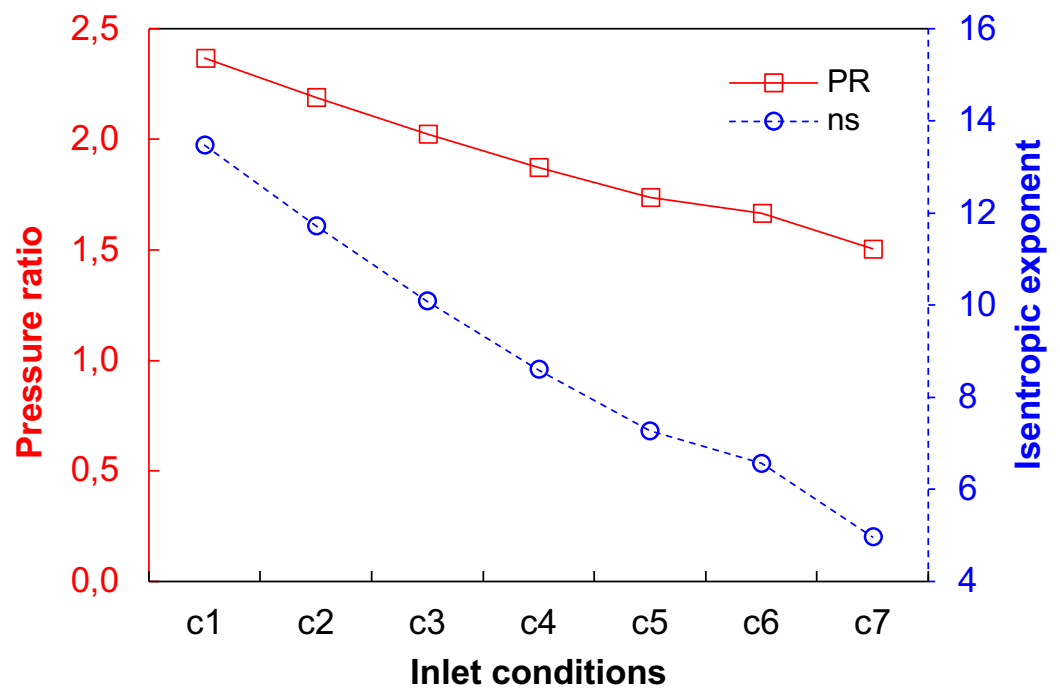

Fig 4: Isentropic exponent $\left(n_{s}\right)$ and pressure ratio $(P R)$ at the reference conditions of $27^{\circ} \mathrm{C}$ and 8.40 MPa.

\subsubsection{Pressure ratio correction}

Let's assume that the compression process within the compressor is characterized by a pressure ratio $\Pi$, an efficiency $\eta$, and an isentropic exponent $\bar{n}_{s}$. The total enthalpy rise reads:

$$
\Delta H_{t}=\frac{1}{\eta} \frac{\bar{n}_{s}}{\bar{n}_{s}-1} r Z_{t} T_{t}\left(\Pi^{\frac{\bar{n}_{s}-1}{\bar{n}_{s}}-1}\right)
$$

in which the "bar" sign above the isentropic exponent implies that it is a variable of a process rather than a property of the fluid at a specific state. However, only the inlet conditions are specified when using the performance maps, and thus this process variable remains unknown. For the sake of simplicity, let's assume that it could be replaced by the isentropic exponent of fluid at the compressor inlet $\left(n_{s}\right)$.

Equation (7-32) can be normalized to formulate the corrected enthalpy rise as follow:

$$
\Delta H_{a d}=\frac{\Delta H_{t}}{n_{s} r Z_{t} T_{t}}=\frac{1}{\eta} \frac{1}{n_{s}-1}\left(\Pi^{\frac{n_{s}-1}{n_{s}}}-1\right)
$$

As the variation of the corrected enthalpy rise and efficiency is small at a given set of dimensionless mass flowrate and speed, the following balance can be established between reference and actual conditions (denoted by the subscripts $r e f$ and $a$, respectively): 


$$
\frac{1}{n_{s}-1}\left(\Pi^{\frac{n_{s}-1}{n_{s}}}-1\right)=\left.\frac{1}{n_{s}-1}\left(\Pi^{\frac{n_{s}-1}{n_{s}}}-1\right)\right|_{r e f}
$$

Finally, the corrected pressure ratio, i.e. pressure ratio at the reference condition, is a function of the actual pressure ratio and the isentropic exponents at both actual and reference conditions.

$$
\Pi_{c}=f\left(\Pi, n_{s, a}, n_{s, r e f}\right)
$$

Its explicit formulation is given as follow:

$$
\Pi_{c}=\left[\frac{n_{s, r e f}-1}{n_{s, a}-1}\left(\Pi_{a}^{\frac{n_{s, a}-1}{n_{s, a}}}-1\right)+1\right]^{\frac{n_{s, r e f}}{n_{s, r e f}-1}}
$$

The dependence of the corrected pressure ratio on the actual isentropic exponent is plotted in Fig 5 for some actual pressure ratio at the reference isentropic exponent of 11.7 (i.e. at $27^{\circ} \mathrm{C}$ and $8.40 \mathrm{MPa}$ ). It comes out that, in the phase of generating the performance maps, smaller actual isentropic exponent results in higher corrected pressure ratio. When using the performance maps, an inlet condition featuring a smaller isentropic exponent will have lower pressure ratio compared to the reference value.

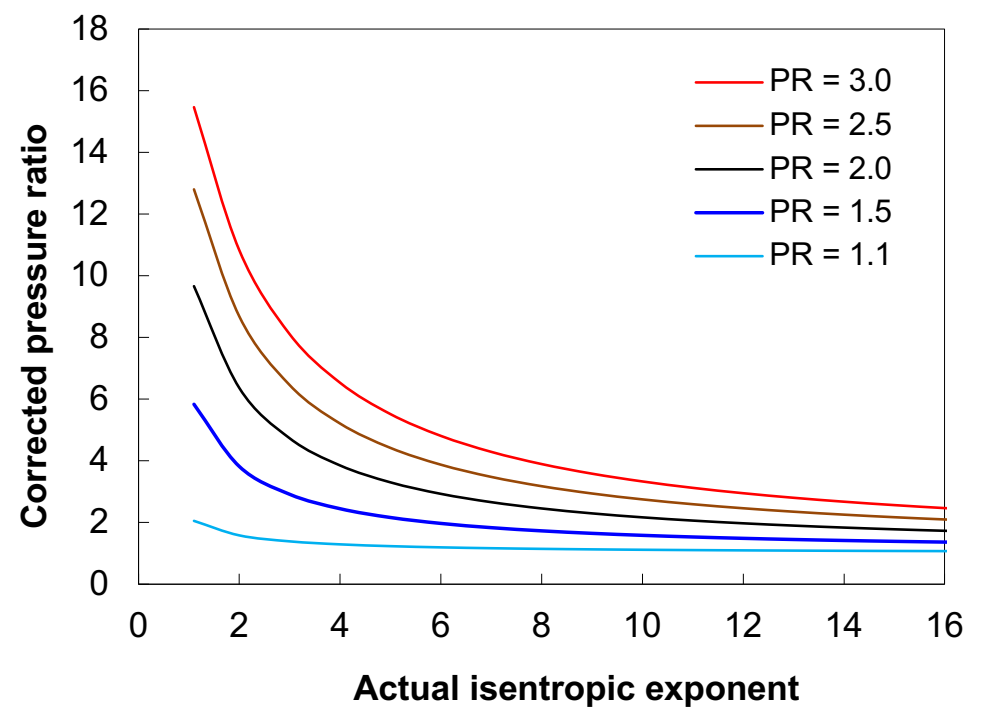

Fig 5: Dependence of the corrected pressure ratio on the isentropic exponent at various pressure ratios

Applying this correction to the pressure ratios achieved in the previous CFD simulations, the corrected pressure ratios are now very close to the one at the reference condition (case c2). The maximum error found on the outlet pressure is below $0.03 \mathrm{MPa}$ (cf. Table 15), indicating the relevancy of the above correction formulation.

Table 15: Corrected pressure ratio from the previous CFD simulations cases.

\begin{tabular}{|l|c|c|c|c|c|c|c|}
\hline Case & $\mathrm{c} 1$ & $\mathrm{c} 2$ & $\mathrm{c} 3$ & $\mathrm{c} 4$ & $\mathrm{c} 5$ & $\mathrm{c} 6$ & $\mathrm{c} 7$ \\
\hline$n_{s}$ & 13.5 & 11.7 & 10.1 & 8.6 & 7.3 & 6.6 & 5.0 \\
\hline$P R_{a}$ & 2.367 & 2.189 & 2.022 & 1.872 & 1.737 & 1.666 & 1.505 \\
\hline$P R_{c}$ & 2.190 & 2.189 & 2.186 & 2.186 & 2.184 & 2.185 & 2.184 \\
\hline
\end{tabular}




\begin{tabular}{|l|l|l|l|l|l|l|l|}
\hline$\Delta P_{\text {out }}(\mathrm{MPa})$ & -0.01 & 0.00 & 0.02 & 0.02 & 0.03 & 0.02 & 0.02 \\
\hline
\end{tabular}

The next step is to examine the present approach at the inlet conditions that revealed the limitations of the BNI approach, i.e. the ones that feature very large change in the specific heat ratio (cf. Table 13). Maximum errors of $0.6 \%$ and 0.9 point have been found for the corrected enthalpy rise and efficiency, respectively (c.f. Table 16). As such, a very good consistency has been achieved on the performance parameters, indicating the improvement of this approach compared to the BNI one.

Table 16: Performance comparison between the IGZ and BNI correction approaches at the corrected mass flowrate and speed of $436 \mathrm{~kg} / \mathrm{s}$ and $3087 \mathrm{rpm}$ with respect to the reference conditions of $34.0^{\circ} \mathrm{C}$ and $7.86 \mathrm{MPa}$.

\begin{tabular}{|c|c|c|c|c|c|c|c|c|c|}
\hline \multirow[b]{2}{*}{$\begin{array}{l}\mathrm{T}_{\mathrm{t}, \mathrm{in}} \\
\left({ }^{\circ} \mathrm{C}\right)\end{array}$} & \multirow[b]{2}{*}{$\begin{array}{c}\mathrm{P}_{\mathrm{t}, \mathrm{in}} \\
(\mathrm{MPa})\end{array}$} & \multirow[b]{2}{*}{$\gamma$} & \multirow[b]{2}{*}{$n_{s}$} & \multicolumn{3}{|c|}{ BNI approach } & \multicolumn{3}{|c|}{ Present approach } \\
\hline & & & & $\begin{array}{c}\Delta \mathrm{H}_{\mathrm{c}} \\
(\mathrm{kJ} / \mathrm{kg})\end{array}$ & PR & $\eta(\%)$ & $\begin{array}{c}\Delta \mathrm{H}_{\mathrm{c}} \\
(\mathrm{kJ} / \mathrm{kg})\end{array}$ & $\mathrm{PR}_{\mathrm{c}}$ & $\eta(\%)$ \\
\hline 34.0 & 6.00 & 2.41 & 1.3 & 3.56 & 1.141 & 90.13 & 3.25 & 1.173 & 92.58 \\
\hline 27.0 & 9.00 & 3.46 & 12.6 & 3.41 & 1.144 & 91.57 & 3.27 & 1.173 & 92.56 \\
\hline 35.0 & 8.05 & 24.5 & 1.9 & 3.15 & 1.171 & 92.22 & 3.27 & 1.173 & 91.70 \\
\hline \multicolumn{4}{|c|}{ Maximum discrepancy } & $12 \%$ & 0.030 & 2.09 & $0.6 \%$ & 0.000 & 0.88 \\
\hline
\end{tabular}

\subsection{Performance maps approach qualification}

The final task consists in extending the application of the above correction approach (cf. Eqs $8,9,12$ and 17) to a wider range of inlet conditions as well as of mass flowrate and shaft speed. An extensive number of simulations has been carried out for such a purpose. The inlet conditions of temperature and pressure are recapitulated in Fig 6, showing a concentration in the subcritical liquid and supercritical region. The plots of the corrected pressure ratio, corrected enthalpy rise and efficiency are reported in Fig 7, Fig 8 and Fig 9, respectively. Each point shown in these plots is constituted of 3 to 7 sets of inlet conditions; over 100 points have been used to establish these charts. The consistency of the these performance parameters at various corrected speed and mass flowrate regardless the change of the inlet conditions confirm the relevancy of this performance maps approach for the sc- $\mathrm{CO}_{2}$ compressor.

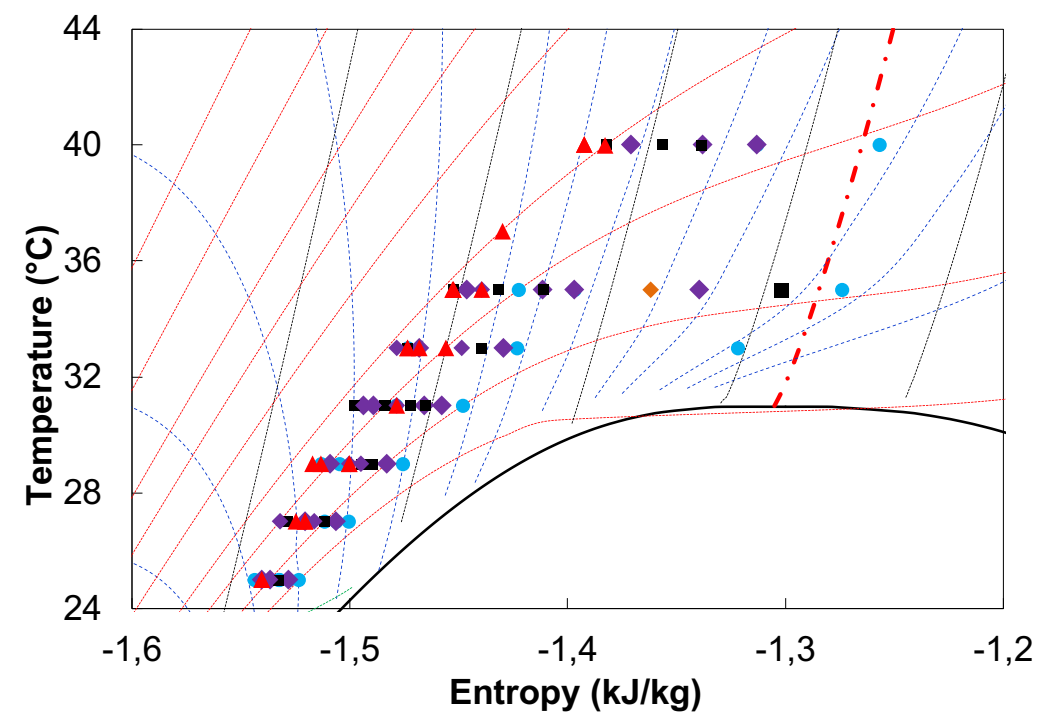

Fig 6: Distribution of inlet conditions used to qualify the compressor performance maps approach. 


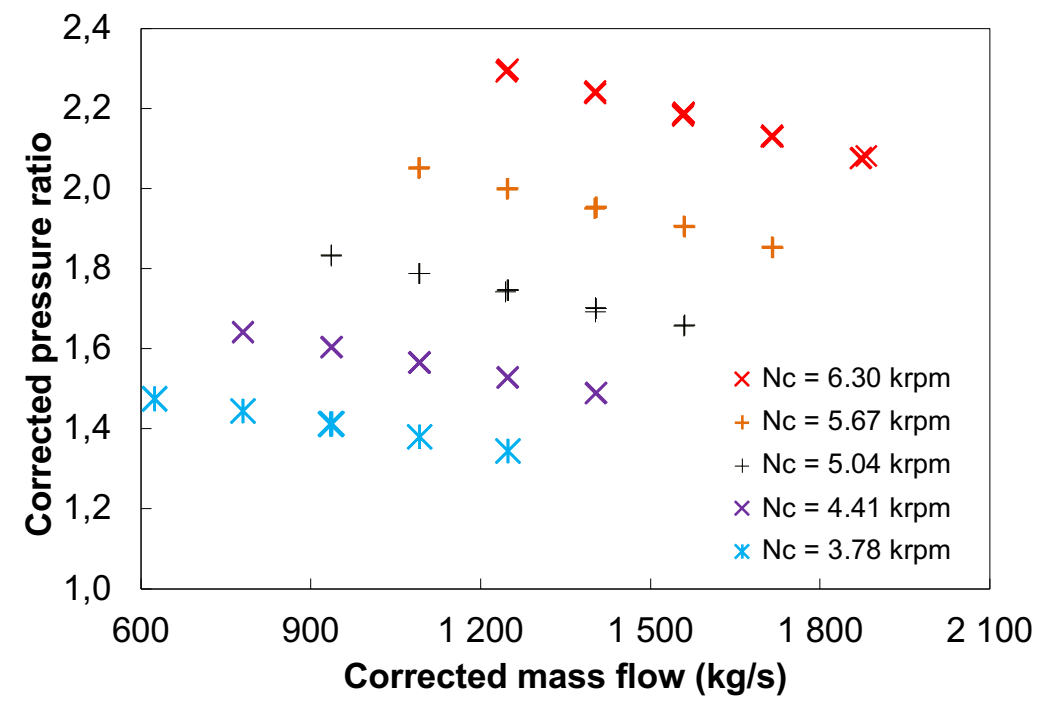

Fig 7: Chart of corrected pressure ratio at the reference conditions of $27^{\circ} \mathrm{C}$ and $8.4 \mathrm{MPa}$.

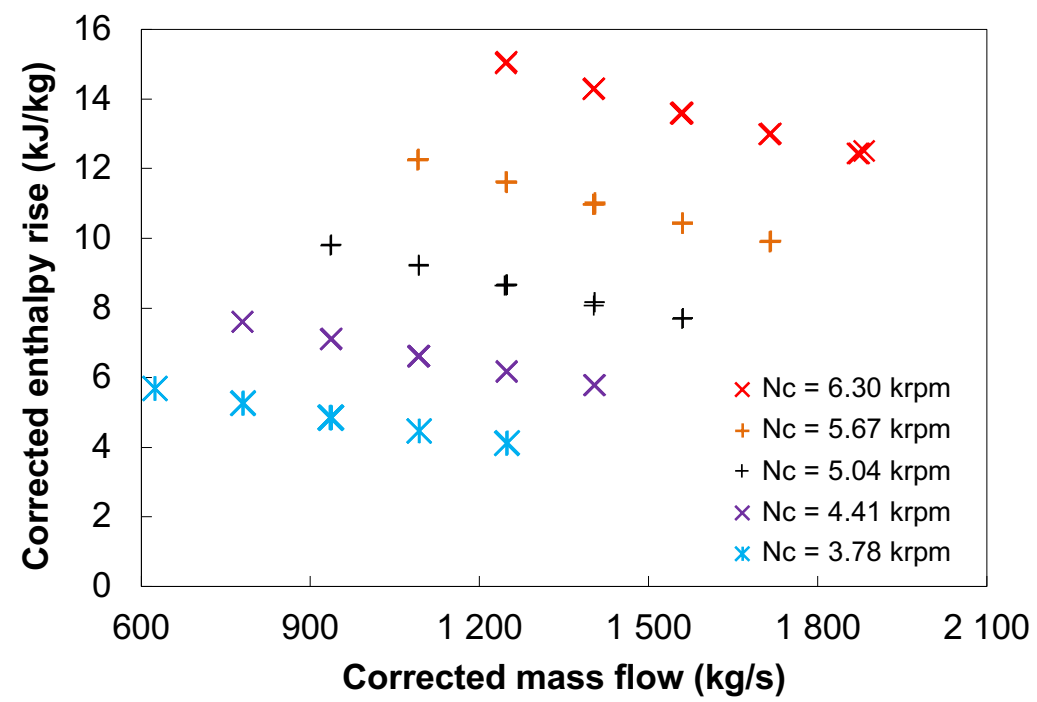

Fig 8: Chart of corrected enthalpy rise at the reference conditions of $27^{\circ} \mathrm{C}$ and $8.4 \mathrm{MPa}$.

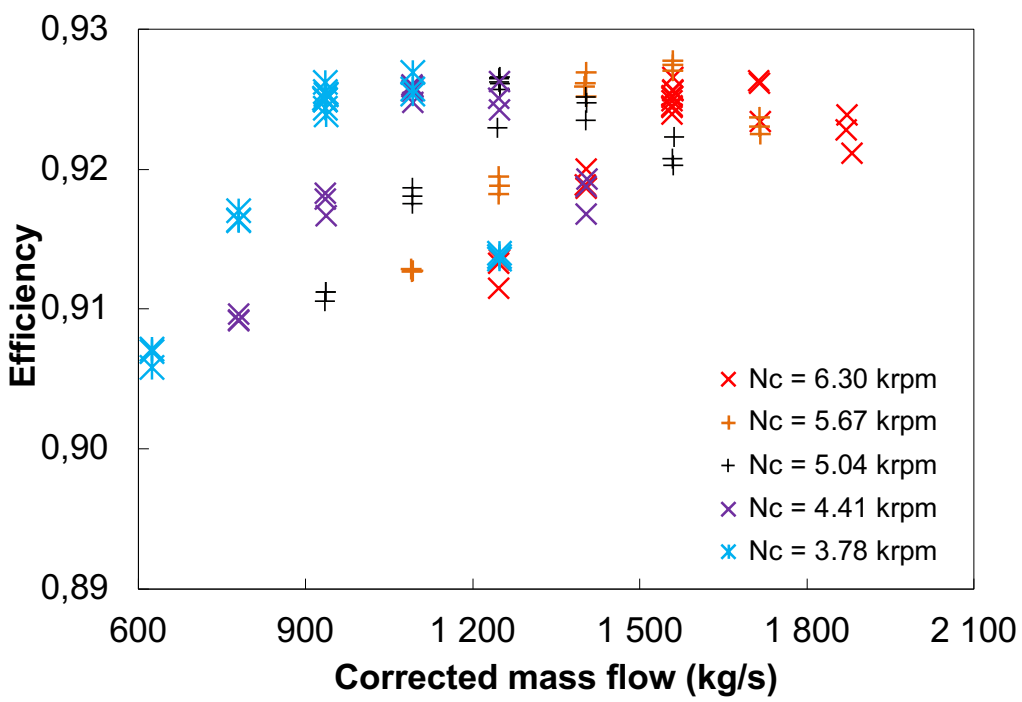

Fig 9: Chart of compressor efficiency at the reference conditions of $27^{\circ} \mathrm{C}$ and $8.4 \mathrm{MPa}$. 


\section{Conclusions}

CFD simulations have been performed on the small scale TiTech sc- $\mathrm{CO}_{2}$ compressor and the numerical results have been confronted with the experimental data. Measurement uncertainties have been found to have strong implications on the reliability of the compressor performance so that this latter could not be confronted with the simulation results. Nevertheless, a very good agreement regarding the temperatures and pressures at the compressor inlet and outlet has been achieved, promisingly suggesting the qualification of the CFD technique.

Moving onto a full scale compressor design, CFD simulations have disclosed the significant decrease in the static temperature and static pressure in the local area of flow acceleration. This fact indicates a high potential for appearing the two-phase condition inside the compressor. To avoid such a concern, it is recommended that the compressor inlet conditions should be chosen at the lowest temperature that can accommodate the heat sink temperature. In addition, the sc- $\mathrm{CO}_{2}$ compressor should also be sized at low inlet velocity and shaft speed, keeping the maximum velocity of the flow nearby the impeller leading edge below a certain limit. In that context, the actual impact of the two-phase flow appearance inside the sc- $\mathrm{CO}_{2}$ compressor in terms of performance and mechanical consequences on structure deserves deep analytical investigation, in particular since it was reported in the literature that compressor operation in two phase flow yielded in fact no dysfunctional behavior nearby the critical point.

A revision of some previous performance maps approaches has been carried out, emphasizing their limits when applied to the $\mathrm{sc}-\mathrm{CO}_{2}$ compressor. An approach that can accommodate the real gas behavior of the working fluid has been proposed on the basis of some key dimensionless numbers of the turbomachinery problem. Using CFD results on a full scale compressor, these 3 dimensional maps have been showed to be able to consistently represent and reliably predict the $\mathrm{sc}-\mathrm{CO}_{2}$ compressor performance over a large operation range from the subcritical liquid to supercritical phase. Such an approach is seen to ease the experimental validation compared to a 4 dimensional approach, as well as to simplify the numerical treatment of performance maps in a system code.

\section{Acknowledgements}

The present study includes the result of a "Study of $\mathrm{Na}$ cooled fast reactor supercritical $\mathrm{CO}_{2}$ turbine system" entrusted to Tokyo Institute of Technology by the Ministry of Education, Culture, Sports, Science and Technology of Japan (MEXT). We thank Dr. Kisohara (Japan Atomic Energy Agency) for his strong support to promote the TiTech-CEA "Cooperation for computer analysis of the sc- $\mathrm{CO}_{2}$ compressor loop experimental data". 


\section{References}

Alpy, N., Haubensack, D., Simon, N., Gicquel, L., Rodriguez, G., Cachon, L., 2011. Gas Cycle Testing Opportunity with ASTRID, the French SFR Prototype, in: Proceeding of the 2011 Supercritical CO2 Power Cycle Symposium. Boulder, Colorado, U.S.

Angelino, G., 1968. Carbon Dioxide Condensation Cycles For Power Production. Journal of Engineering for Power 90, 287 - 295.

Aritomi, M., Ishizuka, T., Muto, Y., Tsuzuki, N., 2011. Performance Test Results of a Supercritical CO2 Compressor Used in a New Gas Turbine Generating System. Journal of Power and Energy Systems 5, 45-59.

Baltadjiev, N., Lettieri, C., Spakovszky, Z., 2014. An Investigation of Real Gas Effects in Supercritical CO2 Centrifugal Compressors. p. V03BT36A011. doi:10.1115/GT2014-26180

Chen, Y., 2011. Thermodynamic Cycles using Carbon Dioxide as Working Fluid : CO2 Transcritical Power Cycle Study (Dissertation). KTH Royal Institute of Technology.

Dostal, V., 2004. A Supercritical Carbon Dioxide Cycle for Next Generation Nuclear Reactors (Doctoral thesis). Massachusetts Institute of Technology.

Feher, E.G., 1968. The Supercritical Thermodynamic Power Cycle. Energy Conversion 8, 85-90.

Floyd, J., Alpy, N., Moisseytsev, A., Haubensack, D., Rodriguez, G., Sienicki, J., and Avakian G., 2013. A Numerical Investigation of the sCO2 Recompression Cycle off-design Behaviour, Coupled to a Sodium-cooled Fast Reactor, for Seasonal Variation in the Heat Sink Temperature, Nuclear Engineering and Design, vol. 260, 78-92.

Glassman, A.J., 1972. Turbine Design and Application. National Aeronautics and Space Administration, Scientific and Technical Information Office, Washington.

Hejzlar, P., Dostal, V., Driscoll, M.. J., Dumaz, P., Poullennec, G., Alpy, N., 2006. Assessment of Gas-cooled Fast Reactor with Indirect Supercritical CO2 Cycle. Nuclear Engineering and Technology 38, 436-446.

Hiroaki, T., Ayao, T., Masaru, H., Nuchi, N., 1973. Effects of buoyancy and of acceleration owing to thermal expansion on forced turbulent convection in vertical circular tubes-criteria of the effects, velocity and temperature profiles, and reverse transition from turbulent to laminar flow. International Journal of Heat and Mass Transfer 16, 1267-1288. doi:10.1016/00179310(73)90135-X

Ishiyama, S., Muto, Y., Kato, Y., Nishio, S., Hayashi, T., Nomoto, Y., 2008. Study of Steam, Helium and Supercritical CO2 Turbine Power Generations in Prototype Fusion Power Reactor. Progress in Nuclear Energy 50, 325 - 332.

Karthik, T.S.D., 2011. Turbulence Models and their Applications. Presented at the 10th Indo German Winter Academy 2011.

Kidnay, A.J., Parrish, W.R., McCartney, D.G., 2011. Fundamentals of Natural Gas Processing, Second Edition. CRC Press.

Kurganov, V.A., Kaptil'ny, A.G., 1992. Velocity and enthalpy fields and eddy diffusivities in a heated supercritical fluid flow. Experimental Thermal and Fluid Science 5, 465-478. doi:10.1016/0894-1777(92)90033-2

Liu, Z., Hill, D.L., 2000. Issues surrounding multiple frames of reference models for turbo compressor applications, in: Proceedings of 15th International Compressor Engineering Conference at Purdue University. West Lafayette.

Lüdtke, K.H., 2004. Process Centrifugal Compressors: Basics, Function, Operation, Design, Application. Springer. 
Moisseytsev, A., Sienicki, J.J., 2010. Analysis of SNL Compressor Tests (No. ANL-GenIV-136). Argonne National Laboratory.

Moisseytsev, A., Sienicki, J.J., 2006. Development of a Plant Dynamics Computer Code for Analysis of a Supercritical Carbon Dioxide Brayton Cycle Energy Converter Coupled to a Natural Circulation Lead-Cooled Fast Reactor (No. ANL-06/27). Argonne National Laboratory.

Muto, Y., Nitawaki, T., Kato, Y., 2003. Comparative Design Study of Carbon Dioxide Gas Turbine and Helium Turbine for HTGR Power Plant. Presented at the International Congress on Advanced Nuclear Power Plants (ICAPP '03), Cordoba, Spain.

Noall, J.S., Pasch, J.J., 2014. Achievable Efficiency and Stability of Supercritical CO2 Compression Systems, in: Supercritical CO2 Power Cycle Symposium. Pittsburgh, Pennsylvania.

Pecnik, R., Rinaldi, E., Colonna, P., 2012. Computational Fluid Dynamics of a Radial Compressor Operating With Supercritical CO2. J. Eng. Gas Turbines Power 134, 122301-122301. doi:10.1115/1.4007196

Pham, H.S., Alpy, N., Ferrasse, J.H., Boutin, O., Quenaut, J., Tothill, M., Haubensack, D., Saez, M., 2015. Mapping of the thermodynamic performance of the supercritical CO2 cycle and optimisation for a small modular reactor and a sodium-cooled fast reactor. Energy 87. doi:10.1016/j.energy.2015.05.022

Rinaldi, E., Pecnik, R., Colonna, P., 2013. Steady State CFD Investigation of a Radial Compressor Operating With Supercritical CO2. ASME, p. V008T34A008. doi:10.1115/GT2013-94580

Roberts, S.K., Sjolander, S.A., 2005. Effect of the Specific Heat Ratio on the Aerodynamic Performance of Turbomachinery. Journal of Engineering for Gas Turbines and Power 127, 773. doi:10.1115/1.1995767

Sanchez, D., Chacartegui, R., Jimenez-Espadafor, F., Sanchez, T., 2009. A New Concept for High Temperature Fuel Cell Hybrid Systems Using Supercritical Carbon Dioxide. Journal of Fuel Cell Science and Technology 6.

Sienicki, J.J., 2005. SSTAR Lead-cooled, Small Modular Fast Reactor with Nitride Fuel.

Span, R., Wagner, W., 1996. A New Equation of State for Carbon Dioxide Covering the Fluid Region from the Triple-Point Temperature to $1100 \mathrm{~K}$ at Pressures up to $800 \mathrm{MPa}$. Journal of Physical and Chemical Reference Data 25, 1509.

Turchi, C.S., Ma, Z., Neises, T.W., Wagner, M.J., 2013. Thermodynamic Study of Advanced Supercritical Carbon Dioxide Power Cycles for Concentrating Solar Power Systems. Journal of Solar Energy Engineering 135, 041007.

Vilim, R.B., 2010. A One-Dimensional Compressor Model for Super-Critical Carbon Dioxide Applications, in: Proceedings of ICAPP'10. San Diego, CA, USA.

Wahl, G.D., 2009. Efficiency Uncertainty of a Turbine Driven Compressor in a Supercritical CO2 Brayton Cycle. Presented at the Proceeding of Supercritical CO2 Power Cycle Symposium.

Wright, S.A., Conboy, T.M., Radel, R.F., Rochau, G.E., 2011. Modeling and experimental results for condensing supercritical $\mathrm{CO} 2$ power cycles.

Wright, S.A., Radel, R.F., Vernon, M.E., Pickard, P.S., Rochau, G.E., 2010. Operation and analysis of a supercritical CO2 Brayton cycle.

Yoon, H.J., Ahn, Y., Lee, J.I., Addad, Y., 2012. Potential Advantages of Coupling Supercritical CO2 Brayton Cycle to Water-cooled Small and Medium Size Reactor. Nuclear Engineering and Design 245, $223-232$. 